\title{
The Effectiveness of Public, Catholic, and Non-Religious Private Schools in Chile's Voucher System
}

\author{
PATRICK J. McEWAN*
}

Abstract In 1980, Chile began financing public and most private schools with vouchers. This paper uses 1997 data on over 150000 Chilean eighth-graders to compare Spanish and mathematics achievement in six types of public and private schools, including voucher schools operated by Catholic and non-religious institutions. Initial findings suggest that Catholic voucher schools have a small advantage over most public schools, once student and peer attributes are controlled for. There is no important difference in achievement between public and non-religious voucher schools, most of which were created in direct response to the 1980 reforms. In some cases, it appears that non-religious voucher schools produce slightly lower achievement than public schools. Accounting for selection bias reduces any private school advantages (or widens their disadvantages), although these estimates are not sufficiently precise to convincingly reject the null hypothesis of no selection bias.

\section{Introduction}

How much does the average student stand to gain (or lose) by transferring from a public to a private school? Most of the empirical research on the topic is, by necessity, non-experimental. It compares the outcomes of students that have chosen to attend private and public schools, while controlling for other variables ('socioeconomic status') that are correlated with school type and outcomes. ${ }^{1}$ More recently, small-scale experiments in the US have compared outcomes of students who were awarded private school vouchers through randomized lotteries. ${ }^{2}$

This empirical evidence is often used to infer the potential impact of a largescale implementation of private school vouchers. If current private schools are effective, reason some, then newly created private schools should be just as effective. But there is good reason to be cautious about making this analytical leap. In the US, most private schools are non-profit and operated by the Catholic church. A voucher plan would encourage the creation of new schools, many profit maximizing and non-religious. These are likely to differ greatly in their objectives, resources, and

* Patrick J. McEwan, University of Illinois at Urbana-Champaign, 360 Education Building, 1310 South Sixth Street, Champaign, IL 61820, USA. Tel: + 1217333 9865; E-mail: mcewan@uiuc.edu. 
constraints, but just how much is little explored. Derek Neal (1998, p. 84) concludes his recent literature review by stating that

... we cannot confidently expect positive outcomes for [voucher] program participants if the program is large in scale ... Large school voucher programs would likely mean the expansion of many existing private schools and the entry of many new private schools. How would this expansion and entry affect the quality of private schools or the quality of remaining public schools? We do not know, and available data shed little light on this question.

One solution is to examine a country where vouchers have been implemented on a large scale and where many new private schools have been created. In 1980, Chile's military government began funding public and most private schools according to their monthly enrollments and a fixed voucher. The supply of private schools quickly responded and their enrollments increased sharply. While many voucher schools are operated by the Catholic church, the majority are operated by private entrepreneurs and were created in direct response to the 1980 reforms. This paper compares the achievement of eighth-graders in many school types, including public, Catholic voucher, and non-religious voucher schools.

This is not the first paper to compare private and public achievement in Chile (Aedo \& Larranaga, 1994; Parry, 1996, 1997c; Aedo, 1998; McEwan \& Carnoy, 2000). But the present analysis is distinguished by four features. First, it uses student-level data, unlike prior studies that were forced to use data averaged to the school level. Second, it uses rich background data on students and their peers, collected in a parent questionnaire administered for the first time in 1997. Third, it attempts to test for selection bias induced by the non-random allocation of students across school types. Fourth, it considers many categories of public and private schools, including Catholic and non-religious voucher schools; prior analysts have generally used a single category to describe all private voucher schools.

This paper proceeds in the following manner. The next section reviews some background on Chile's school system and describes the school types that will serve as the key analytical categories. The third section sets forth the empirical strategy that will be used to compare student achievement in public and private schools, and describes the data that will be used to implement it. The fourth section presents and interprets several empirical analyses, while the final section summarizes and concludes.

\section{Background on Chile}

In 1980, Chile's military government initiated a two-pronged reform. ${ }^{3}$ First, it transferred responsibility for public school management from the Ministry of Education to local municipalities. Once transferred to municipalities, most schools were placed under the control of a Departmento de Administración de la Educación Municipal (DAEM) and others under a quasi-autonomous 'corporation'. ${ }^{4}$ Second, the government altered how public and most private schools were financed. The Ministry of Education began disbursingmonthly payments to municipalities based on a fixed voucher multiplied by the number of students enrolled in their schools. Private schools received equivalent per-student payments if they did not charge tuition. The law established a base voucher level, which varies according to several factors. ${ }^{5}$ 
Table 1. A taxonomy of private and public schools in Chile

\begin{tabular}{|c|c|c|}
\hline School type & Management & Financing \\
\hline Public DAEM & $\begin{array}{l}\text { Departmento de Administración de la } \\
\text { Educación Municipal; part of municipal } \\
\text { bureaucracy }\end{array}$ & $\begin{array}{l}\text { National vouchers; municipal } \\
\text { contributions; Regional Development } \\
\text { Fund (infrastructure); private } \\
\text { contributions }^{\text {a }}\end{array}$ \\
\hline Public Corporation & $\begin{array}{l}\text { Municipal corporation; quasi- } \\
\text { autonomous from municipal } \\
\text { bureaucracy }\end{array}$ & $\begin{array}{l}\text { National vouchers; municipal } \\
\text { contributions; Regional Development } \\
\text { Fund (infrastructure); private } \\
\text { contributions }^{\text {a }}\end{array}$ \\
\hline Catholic voucher & $\begin{array}{l}\text { Branches of Catholic church, including } \\
\text { religious orders, parishes, and the } \\
\text { Archdiocese }\end{array}$ & $\begin{array}{l}\text { National vouchers; private } \\
\text { contributions }^{\mathrm{a}} \text {; church contributions }{ }^{\mathrm{b}}\end{array}$ \\
\hline Protestant voucher & $\begin{array}{l}\text { Protestant churches, including } \\
\text { Methodist, Baptist, Seventh-Day } \\
\text { Adventist, Lutheran, and others not } \\
\text { affiliated with specific denominations }\end{array}$ & $\begin{array}{l}\text { National vouchers; private } \\
\text { contributions }^{\mathrm{a}} \text {; church contributions }{ }^{\mathrm{b}}\end{array}$ \\
\hline Non-religious voucher & $\begin{array}{l}\text { For-profit businesses; non-profit } \\
\text { foundations; individuals }\end{array}$ & $\begin{array}{l}\text { National vouchers; private } \\
\text { contributions }^{\mathrm{a}}\end{array}$ \\
\hline Private non-voucher & $\begin{array}{l}\text { Catholic church; Protestant churches; } \\
\text { for-profit businesses; non-profit } \\
\text { foundations; individuals }\end{array}$ & $\begin{array}{l}\text { Private contributions } \mathrm{s}^{\mathrm{a}} \text {; church } \\
\text { contributions }^{\mathrm{b}}\end{array}$ \\
\hline
\end{tabular}

\footnotetext{
${ }^{\text {a }}$ Private contributions include tuition payments, Parent-Center fees, private fundraising such as raffles, and donations from the private sector.

${ }^{\mathrm{b}}$ Church contributions include contributed services of church personnel and monetary and in-kind church donations.
}

The reform sparked a massive redistribution of enrollments across private and public schools, as well as the creation of many new private schools. Between 1981 and 1996, enrollments in private voucher schools expanded from $15 \%$ to around $33 \%$ of the total; most of these gains at the expense of public school enrollments. Within the category of private voucher schools, there is a great deal of heterogeneity. Prior to the 1980 reforms, when many private schools already received partial government financing, about one-half were managed by the Catholic church, and the rest by non-religious foundations or Protestant churches (Espinola, 1993). A flood of new private voucher schools, mainly non-religious and for-profit, entered the market following the 1980 reforms (Aedo, 1996). The empirical analyses of this paper will always distinguish between religious and nonreligious voucher schools. Table 1 provides brief descriptions of the management and financing of six types of schools: two public (DAEM and corporation) and four private (Catholic voucher, Protestant voucher, non-religious voucher, and non-voucher private schools).

Table 2 shows how primary schools and enrollments are distributed across these six school types. In urban areas, and even more so in rural areas, the majority of schools are still public. ${ }^{6}$ Private voucher schools account for around $26 \%$ of primary schools (grades $1-8$ ) and $33 \%$ of primary enrollments. Most of 
Table 2. Distribution of primary schools and students across school types, 1996

\begin{tabular}{|c|c|c|c|c|c|c|}
\hline & \multicolumn{3}{|c|}{ Percent of schools } & \multicolumn{3}{|c|}{ Percent of enrollment } \\
\hline & $\begin{array}{l}\text { Total } \\
(\%)\end{array}$ & $\begin{array}{c}\text { Urban } \\
(\%)\end{array}$ & $\begin{array}{c}\text { Rural } \\
(\%)\end{array}$ & $\begin{array}{l}\text { Total } \\
(\%)\end{array}$ & $\begin{array}{c}\text { Urban } \\
(\%)\end{array}$ & $\begin{array}{c}\text { Rural } \\
(\%)\end{array}$ \\
\hline Public DAEM & 55.2 & 31.5 & 74.6 & 40.0 & 34.7 & 70.5 \\
\hline Public corporation & 12.3 & 15.5 & 9.6 & 18.5 & 19.4 & 13.4 \\
\hline Catholic voucher & 4.6 & 9.3 & 0.8 & 10.3 & 11.6 & 2.8 \\
\hline Protestant voucher & 1.2 & 1.9 & 0.6 & 1.5 & 1.4 & 1.9 \\
\hline Non-religious voucher & 19.7 & 26.4 & 14.3 & 21.4 & 23.3 & 10.9 \\
\hline Private non-voucher & 7.1 & 15.5 & 0.2 & 8.3 & 9.6 & 0.6 \\
\hline Total & 100 & 100 & 100 & 100 & 100 & 100 \\
\hline Number of schools or students & 8393 & 3779 & 4614 & 2015867 & 1716641 & 299226 \\
\hline
\end{tabular}

Source: Ministerio de Educación and author's calculations.

Notes: Calculations exclude 163 schools (enrolling 49537 students) for which data on rural or private status were incomplete.

these schools are non-religious, but important numbers are also operated by Catholic and Protestant churches. Another 7\% of schools are private but do not accept vouchers; they charge tuition and are considered the elite sector of Chilean education.

\section{Empirical Strategy and Data}

\section{Empirical Strategy}

In this section, I describe an empirical strategy for comparing public and private achievement that will correct for selection bias. I hypothesize that student achievement can be explained by linear models of the following form: ${ }^{7}$

$$
A_{i j}=X_{i j} \beta_{j}+\varepsilon_{i j}
$$

where the achievement $\left(A_{i j}\right)$ of the $i$ th student in the $j$ th school type is a function of independent variables that describe the socio-economic background of the student and the student's peer group $\left(X_{i j}\right)$, as well as an error term $\left(\varepsilon_{i j}\right)$. The $j$ subscript indexes six categories of public and private schools in Chile. Note that the sample is to be split among school types, and separate regression coefficients estimated for each subsample. By doing so the coefficients are not constrained to be equal across school types. $^{8}$

Using the estimates $\beta_{j}$, one can predict the achievement of a 'typical' student in each school type. The choice of this student's characteristics is arbitrary, but researchers often use the mean characteristics of students in the public school alternative, which I denote $\bar{X}$ (for example, Cox \& Jimenez, 1990). Thus, the predicted achievement of the average public school student in the $j$ th school type is:

$$
\hat{A}_{j}=\overline{\mathrm{X}} \hat{\beta}_{j}
$$


The difference in achievement between two school types is estimated by subtracting one prediction from another. The corresponding standard error can also be calculated. ${ }^{9}$ For example, one may estimate the corrected difference in achievement between Catholic voucher schools $(j=3)$ and public DAEM schools $(j=1)$ as $\hat{A}_{3}-\hat{A}_{1}$. This provides an estimate of the expected increase (or decrease) in achievement for the average public school student if she were to attend a Catholic voucher school.

If the independent variables perfectly control for student background, then the preceding method yields unbiased estimates. More likely is that some background variables are imperfectly measured or omitted from regressions. For example, more able or motivated students could select themselves into private schools (or, likewise, schools may select such students from the queue, if school admissions are over-subscribed). Because ability and motivation are unobserved, the relative effectiveness of schools could still be confounded with the background of their students. Towards ameliorating this selection bias, prior research has often applied variants of two-step corrections suggested by Heckman (1979). ${ }^{10}$ These methods presume that a choice is made between two alternatives: private or public.

In the Chilean context, there are six categories of schools available to students. That is, choices are polychotomous, rather than dichotomous. Lee (1983) has devised a two-step selection bias correction for cases where choice is among several alternatives. ${ }^{11}$ Consider the following model:

$$
I^{\star}{ }_{i j}=Z_{i j} \gamma_{j}+v_{i j} \quad(j=1,2,3,4,5,6)
$$

where $I^{\star}{ }_{i j}$ is a latent variable and $Z_{i j}$ is a vector of variables determining school choice for student $i$ in school type $j$. Let $I$ be a polychotomous variable that can take values 1 to 6 ( $I=j$ if the $j$ th school type is chosen). A student attends the $j$ th school type $(I=j)$

$$
\text { iff } I^{\star}>\operatorname{Max}_{j} \star_{s} \quad(s=1,2,3,4,5,6, s \neq j)
$$

Given assumptions about the error term $v_{i j}$, equation (3) can be estimated as a multinomial logit. ${ }^{12}$ Lee (1983) shows how estimates from the multinomial logit can be used to construct a selectivity term for each observation $\left(\lambda_{i j}\right)$, which then becomes an independent variable in achievement regressions:

$$
A_{i j}=X_{i j} \beta_{j}+\lambda_{i j} \theta_{j}+\varepsilon_{i j}
$$

where $\theta_{j}$ is an additional parameter to be estimated. The variable $\lambda_{i j}$ is analogous to the inverse Mills ratio in the common two-step correction proposed by Heckman (1979). It is defined as

$$
\lambda_{i j}=\frac{\phi\left(\Phi^{-1}\left(P_{i j}\right)\right)}{P_{i j}}
$$

where $\phi(.$.$) is the standard normal density, \Phi(.$.$) is the normal distribution$ function, and $P_{i j}$ is the estimated probability (derived from the multinomial logit) that the $i$ th student chooses the $j$ th school type. 
In general, the independent variables that determine achievement $\left(X_{i j}\right)$ in equation (1) are quite similar to those which determine school choice $\left(Z_{i j}\right)$ in equation (3). Measures of parental education, among others, rightly belong in both equations. In the subsequent empirical implementation, however, it is necessary that one or more variables be included in $Z_{i j}$ that are excluded from $X_{i j}$, in order to identify the model. ${ }^{13}$ Recent papers in the US have assumed that family religious affiliation or the local supply of Catholic schools is a determinant of Catholic school choice, but is unrelated to achievement (Evans \& Schwab, 1995; Neal, 1997). I shall posit that an individual's probability of choosing a given school type is affected by the number of schools per square kilometer of each type in her municipality. ${ }^{14}$ All else equal, students are more likely to choose schooling alternatives that are more densely concentrated in their neighborhoods. It is assumed, however, that school densities do not belong in the achievement production function. I shall attempt to corroborate this assumption in the empirical analysis.

\section{Data}

The preceding models are estimated with data from Chile's Sistema de Medición de Calidad de la Educación (SIMCE). Since 1988, SIMCE data are available for the majority of fourth-graders in even years, and eighth-graders in odd years. ${ }^{15}$ However, 1997 is the first year for which student-level achievement data are available, in contrast to school-level averages of prior years. Detailed background data on students are also available for the first time.

Over $90 \%$ of eighth-graders were administered tests of Spanish and mathematics achievement, and their parents responded to additional background questionnaires. Table 3 provides definitions of the dependent and independent variables used in the analysis. The dependent variables, SPANISH and MATH, were originally expressed as the number of items correct on 60-item examinations, although I standardized these variables to a mean of 0 and a standard deviation of 1 .

Several independent variables measure the student's background and socioeconomic status. These include the student's gender (FEMALE), whether the student's mother is Native American (NATAM), years of parental schooling (MTHSCH and FTHSCH), family income (INCOME), and the number of books in the home (BOOKS1-BOOKS8, expressed as a series of dummy variables). Dummy variables are also included for individuals that have missing values for parental schooling (MTHMISS and FTHMISS).

Measures of peer-group characteristics are obtained by averaging individual characteristics over all the students in a given classroom. PCTNATAM gives the percentage of Native Americans in every student's classroom, and AVMTHSCH, AVFTHSCH, and AVINCOME provide measures of the average parental schooling and income in each classroom. Finally, RURAL indicates the relative isolation of the school. Although not reported in subsequent analyses, dummy variables indicating 12 of Chile's regions - relative to the largest Metropolitan Region-are always included.

Table 4 provides descriptive statistics for the 158872 children that comprise the sample, divided by school type. The distribution of children across school types in the sample is similar to the distribution of students in the universe of primary enrollments (see Table 2). About 58\% of students attend public schools (either DAEM or corporation). Another $12 \%$ attend Catholic voucher schools, which represents just over one-third of enrollments in private voucher schools. Other 
Table 3. Variable definitions

SPANISH

MATH

FEMALE

NATAM $^{a}$

$\mathrm{MTHSCH}^{\mathrm{a}}$

MTHMISS

FTHSCH $^{\mathrm{a}}$

FTHMISS

INCOME

BOOKS1-BOOKS8

PCTNATAM

AVMTHSCH

AVFTHSCH

AVINCOME

RURAL
Number of items correct on the eighth-grade Spanish test (standardized to a mean of 0 and a standard deviation of 1)

Number of items correct on the eighth-grade mathematics test (standardized to a mean of 0 and a standard deviation of 1 )

Dummy variable indicating whether student is female

Dummy variable indicating whether student's mother is Native American

Years of schooling of student's mother

Dummy variable indicating whether MTHSCH is missing

Years of schooling of student's father

Dummy variable indicating whether FTHSCH is missing

Monthly family income, divided by 100000

Eight dummy variables indicating the number of books in the family home, ranging from 1 ( 5 or less) to 8 (more than 95). BOOKS 2 is omitted in regressions

Percent of students in classroom with Native American mother

Average schooling of student mothers in classroom

Average schooling of student fathers in classroom

Average monthly household income of students in classroom

Dummy variable indicating whether school is rural

\footnotetext{
a These variables are reported by a parent on a questionnaire.

Note: With one exception, variables are from the Sistema de Medición de la Calidad de la Educación (SIMCE), 1997. RURAL is from Ministry of Education data.
}

enrollments in voucher schools are mostly accounted for by the non-religious category.

In general, males and females are evenly distributed across school types, although girls are somewhat more likely to attend Catholic schools. Native American students are less likely to attend private non-voucher schools, perhaps because the latter are overwhelmingly located in urban areas. On average, public school parents have lower levels of parental schooling, books in the home, and income. Parents in private non-voucher schools have, by far, the highest levels of such attributes. Parents in private voucher schools also have higher levels than parents of children in public school, but the differences are less pronounced.

\section{Empirical Results}

This section proceeds in four steps. First, it describes and interprets estimates of equation (1) that do not control for selection bias. Second, it presents results from a multinomial logit model of school choice that is based on equation (3). Third, it assesses whether the exclusion restrictions used in the selection bias correction are 
Table 4. Variable means and standard deviations

\begin{tabular}{|c|c|c|c|c|c|c|c|}
\hline & $\begin{array}{l}\text { Entire } \\
\text { sample }\end{array}$ & $\begin{array}{l}\text { Public } \\
\text { DAEM }\end{array}$ & $\begin{array}{c}\text { Public } \\
\text { corporation }\end{array}$ & $\begin{array}{l}\text { Catholic } \\
\text { voucher }\end{array}$ & $\begin{array}{l}\text { Protestant } \\
\text { voucher }\end{array}$ & $\begin{array}{l}\text { Non- } \\
\text { religious } \\
\text { voucher }\end{array}$ & $\begin{array}{c}\text { Private } \\
\text { non- } \\
\text { voucher }\end{array}$ \\
\hline SPANISH & $\begin{array}{c}0.00 \\
(1.00)\end{array}$ & $\begin{array}{c}-0.22 \\
(0.98)\end{array}$ & $\begin{array}{c}-0.20 \\
(0.98)\end{array}$ & $\begin{array}{c}0.40 \\
(0.86)\end{array}$ & $\begin{array}{c}-0.07 \\
(0.97)\end{array}$ & $\begin{array}{c}0.01 \\
(0.98)\end{array}$ & $\begin{array}{c}0.84 \\
(0.72)\end{array}$ \\
\hline MATH & $\begin{array}{c}0.00 \\
(1.00)\end{array}$ & $\begin{array}{c}-0.17 \\
(0.96)\end{array}$ & $\begin{array}{c}-0.25 \\
(0.94)\end{array}$ & $\begin{array}{c}0.39 \\
(0.91)\end{array}$ & $\begin{array}{c}-0.18 \\
(0.91)\end{array}$ & $\begin{array}{c}-0.05 \\
(0.97)\end{array}$ & $\begin{array}{c}0.92 \\
(0.82)\end{array}$ \\
\hline Public DAEM & 0.39 & - & - & - & - & - & - \\
\hline Public corporation & 0.19 & - & - & - & - & - & - \\
\hline Catholic voucher & 0.12 & - & - & - & - & - & - \\
\hline Protestant voucher & 0.01 & - & - & - & - & - & - \\
\hline Non-religious voucher & 0.20 & - & - & - & - & - & - \\
\hline Private non-voucher & 0.09 & - & - & - & - & - & - \\
\hline FEMALE & 0.52 & 0.51 & 0.51 & 0.61 & 0.51 & 0.50 & 0.50 \\
\hline NATAM & 0.05 & 0.07 & 0.04 & 0.03 & 0.08 & 0.05 & 0.01 \\
\hline MTHSCH & $\begin{array}{c}9.53 \\
(3.96)\end{array}$ & $\begin{array}{c}8.22 \\
(3.66)\end{array}$ & $\begin{array}{c}8.89 \\
(3.52)\end{array}$ & $\begin{array}{l}10.88 \\
(3.52)\end{array}$ & $\begin{array}{c}9.85 \\
(3.57)\end{array}$ & $\begin{array}{c}9.83 \\
(3.59)\end{array}$ & $\begin{array}{l}14.25 \\
(3.17)\end{array}$ \\
\hline MTHMISS & 0.02 & 0.02 & 0.02 & 0.01 & 0.02 & 0.02 & 0.01 \\
\hline FTHSCH & $\begin{array}{c}9.83 \\
(4.35)\end{array}$ & $\begin{array}{c}8.33 \\
(4.01)\end{array}$ & $\begin{array}{c}9.25 \\
(3.86)\end{array}$ & $\begin{array}{l}11.12 \\
(3.90)\end{array}$ & $\begin{array}{l}10.20 \\
(3.94)\end{array}$ & $\begin{array}{l}10.17 \\
(3.86)\end{array}$ & $\begin{array}{l}15.17 \\
(3.56)\end{array}$ \\
\hline FTHMISS & 0.03 & 0.03 & 0.03 & 0.02 & 0.03 & 0.03 & 0.02 \\
\hline INCOME & $\begin{array}{c}2.83 \\
(3.87)\end{array}$ & $\begin{array}{c}1.57 \\
(1.79)\end{array}$ & $\begin{array}{c}1.99 \\
(1.93)\end{array}$ & $\begin{array}{c}2.81 \\
(2.66)\end{array}$ & $\begin{array}{c}2.30 \\
(2.21)\end{array}$ & $\begin{array}{c}2.47 \\
(2.36)\end{array}$ & $\begin{array}{l}11.07 \\
(6.82)\end{array}$ \\
\hline BOOKS1 & 0.12 & 0.19 & 0.12 & 0.05 & 0.09 & 0.07 & 0.00 \\
\hline BOOKS2 & 0.26 & 0.34 & 0.28 & 0.17 & 0.27 & 0.22 & 0.04 \\
\hline BOOKS3 & 0.15 & 0.15 & 0.16 & 0.15 & 0.17 & 0.16 & 0.06 \\
\hline BOOKS4 & 0.12 & 0.10 & 0.13 & 0.14 & 0.13 & 0.14 & 0.10 \\
\hline BOOKS5 & 0.07 & 0.05 & 0.07 & 0.09 & 0.08 & 0.08 & 0.08 \\
\hline BOOKS6 & 0.05 & 0.04 & 0.05 & 0.07 & 0.05 & 0.07 & 0.08 \\
\hline BOOKS7 & 0.03 & 0.02 & 0.03 & 0.05 & 0.04 & 0.04 & 0.06 \\
\hline BOOKS8 & 0.20 & 0.11 & 0.15 & 0.27 & 0.18 & 0.22 & 0.58 \\
\hline RURAL & 0.10 & 0.17 & 0.09 & 0.03 & 0.19 & 0.04 & 0.01 \\
\hline PCTNATAM & $\begin{array}{l}5.08 \\
(9.42)\end{array}$ & $\begin{array}{c}6.78 \\
(11.10)\end{array}$ & $\begin{array}{c}4.60 \\
(6.02)\end{array}$ & $\begin{array}{c}2.93 \\
(4.92)\end{array}$ & $\begin{array}{c}8.36 \\
(13.86)\end{array}$ & $\begin{array}{c}5.16 \\
(10.97)\end{array}$ & $\begin{array}{c}0.64 \\
(2.08)\end{array}$ \\
\hline AVMTHSCH & $\begin{array}{c}9.67 \\
(2.47)\end{array}$ & $\begin{array}{c}8.35 \\
(1.84)\end{array}$ & $\begin{array}{c}9.02 \\
(1.65)\end{array}$ & $\begin{array}{l}11.01 \\
(1.75)\end{array}$ & $\begin{array}{l}10.03 \\
(1.71)\end{array}$ & $\begin{array}{c}9.98 \\
(1.87)\end{array}$ & $\begin{array}{l}14.42 \\
(1.35)\end{array}$ \\
\hline AVFTHSCH & $\begin{array}{l}10.08 \\
(2.71)\end{array}$ & $\begin{array}{c}8.58 \\
(2.03)\end{array}$ & $\begin{array}{c}9.51 \\
(1.78)\end{array}$ & $\begin{array}{l}11.33 \\
(1.90)\end{array}$ & $\begin{array}{l}10.46 \\
(1.87)\end{array}$ & $\begin{array}{l}10.42 \\
(1.93)\end{array}$ & $\begin{array}{l}15.42 \\
(1.57)\end{array}$ \\
\hline AVINCOME & $\begin{array}{c}2.83 \\
(3.10)\end{array}$ & $\begin{array}{c}1.57 \\
(0.78)\end{array}$ & $\begin{array}{c}1.99 \\
(0.81)\end{array}$ & $\begin{array}{c}2.81 \\
(1.33)\end{array}$ & $\begin{array}{c}2.31 \\
(0.82)\end{array}$ & $\begin{array}{c}2.47 \\
(1.21)\end{array}$ & $\begin{array}{l}11.06 \\
(4.62)\end{array}$ \\
\hline$\lambda^{\mathrm{a}}$ & $\begin{array}{c}1.02 \\
(0.54)\end{array}$ & $\begin{array}{c}0.71 \\
(0.32)\end{array}$ & $\begin{array}{c}1.17 \\
(0.35)\end{array}$ & $\begin{array}{c}1.54 \\
(0.37)\end{array}$ & $\begin{array}{c}2.38 \\
(0.50)\end{array}$ & $\begin{array}{c}1.20 \\
(0.45)\end{array}$ & $\begin{array}{c}0.82 \\
(0.79)\end{array}$ \\
\hline Number of individuals & 158872 & 62684 & 30050 & 18442 & 2268 & 31249 & 14179 \\
\hline
\end{tabular}

${ }^{\text {a }}$ See text for definition of $\lambda$.

Note: Standard deviations are not reported for dummy variables. 
valid-that is, whether school densities can properly be excluded from the achievement regressions. Fourth, the estimates from the multinomial logit are used to construct a selectivity term $\left(\lambda_{i j}\right)$. The achievement regressions are re-estimated with $\lambda_{i j}$ as an independent variable in order to assess the importance of selection bias.

\section{Initial Results}

The achievement regressions presented in Table 5 provide estimates of equation (1), using SPANISH as the dependent variable. Results suggest that the Spanish achievement of female students is higher, on average, than that of males. In every school type, the advantage is around 0.2 standard deviations. Native American students score lower, on average, than other students, although coefficients are statistically significant only for public DAEM and Catholic schools. The variables that proxy the socio-economic status of individual students are generally of the expected signs. Parental schooling, particularly that of mothers, is strongly related to Spanish achievement. The number of books in the home, gauged by a series of dummy variables, is also positively associated with achievement. Coefficients on the family income variable, however, are either negative or statistically insignificant. The results for mathematics presented in Table 6 are quite similar, with one exception. The gender gap is reversed, with girls scoring slightly lower in every school type except private non-voucher schools (although coefficients are only statistically significant in the case of the public DAEM and non-religious voucher schools).

Among the attributes of student peer groups, the percentage of Native American students in a classroom is negatively related to achievement, although not strongly (a 10 percentage point increase leads to an average decline of around 0.04 standard deviations in the Spanish achievement among public DAEM students). Coefficients on the average schooling of mothers vary between 0.05 and 0.12 across school types when SPANISH is the dependent variable. If average schooling increases by 1.8 years among public DAEM students-a full standard deviation-the average student's achievement rises between 0.09 and 0.22 standard deviations.

The preceding results are suggestive of peer effects, but there is a caveat. Peer variables may be measuring unobserved aspects of individuals. For example, extremely motivated parents may seek out 'better' peer groups for their children. If motivation is omitted from regressions and positively correlated with achievement, then the peer-group effects are probably overstated because they capture aspects of individual or family background. ${ }^{16}$ Nonetheless, the immediate concern of this analysis is the unbiased estimation of private school effects. Peer-group variables, while somewhat difficult to interpret in ordinary least-squares regressions, are still a good means of capturing omitted variation in student background that could bias estimates of private school effects.

Following equation (2), one can predict the achievement of the average public DAEM student in each school type. By subtracting the predicted achievement in public DAEM schools from that of other school types, one can estimate the relative effects of private schools on achievement. These results are presented in Table 7, along with the standard errors of each estimate.

Before turning to the main results, let us consider the 'unadjusted differences' in achievement. These are simply the average difference in student achievement between DAEM students and those of other school types, uncorrected for background variables (I obtained these estimates and standard errors from simple 
Table 5. Spanish achievement regressions without selectivity correction

\begin{tabular}{|c|c|c|c|c|c|c|}
\hline & \multicolumn{6}{|c|}{ Dependent variable: SPANISH } \\
\hline & $\begin{array}{l}\text { Public } \\
\text { DAEM }\end{array}$ & $\begin{array}{c}\text { Public } \\
\text { corporation }\end{array}$ & $\begin{array}{l}\text { Catholic } \\
\text { voucher }\end{array}$ & $\begin{array}{l}\text { Protestant } \\
\text { voucher }\end{array}$ & $\begin{array}{l}\text { Non- } \\
\text { religious } \\
\text { voucher }\end{array}$ & $\begin{array}{c}\text { Private } \\
\text { non- } \\
\text { voucher }\end{array}$ \\
\hline FEMALE & $\begin{array}{l}0.198^{\star \star} \\
(0.016)\end{array}$ & $\begin{array}{l}0.189^{\star \star} \\
(0.016)\end{array}$ & $\begin{array}{l}0.204^{\star \star} \\
(0.020)\end{array}$ & $\begin{array}{l}0.184^{\star \star} \\
(0.049)\end{array}$ & $\begin{array}{l}0.182^{\star \star} \\
(0.015)\end{array}$ & $\begin{array}{l}0.218^{\star \star} \\
(0.018)\end{array}$ \\
\hline NATAM & $\begin{array}{l}-0.117^{\star \star} \\
(0.015)\end{array}$ & $\begin{array}{c}-0.041 \\
(0.025)\end{array}$ & $\begin{array}{c}-0.084^{\star} \\
(0.041)\end{array}$ & $\begin{array}{c}-0.027 \\
(0.069)\end{array}$ & $\begin{array}{c}-0.021 \\
(0.025)\end{array}$ & $\begin{array}{c}-0.060 \\
(0.082)\end{array}$ \\
\hline MTHSCH & $\begin{array}{l}0.023^{\star \star} \\
(0.001)\end{array}$ & $\begin{array}{l}0.020^{\star \star} \\
(0.002)\end{array}$ & $\begin{array}{l}0.018^{\star \star} \\
(0.003)\end{array}$ & $\begin{array}{c}0.021^{\star} \\
(0.008)\end{array}$ & $\begin{array}{l}0.022^{\star \star} \\
(0.002)\end{array}$ & $\begin{array}{l}0.016^{\star \star} \\
(0.003)\end{array}$ \\
\hline MTHMISS & $\begin{array}{c}0.055 \\
(0.028)\end{array}$ & $\begin{array}{c}0.078 \\
(0.045)\end{array}$ & $\begin{array}{c}0.074 \\
(0.059)\end{array}$ & $\begin{array}{c}0.275 \\
(0.202)\end{array}$ & $\begin{array}{c}0.059 \\
(0.046)\end{array}$ & $\begin{array}{c}0.160^{\star} \\
(0.079)\end{array}$ \\
\hline FTHSCH & $\begin{array}{l}0.012^{\star \star} \\
(0.001)\end{array}$ & $\begin{array}{l}0.010^{\star \star} \\
(0.002)\end{array}$ & $\begin{array}{l}0.013^{\star \star} \\
(0.002)\end{array}$ & $\begin{array}{c}0.008 \\
(0.008)\end{array}$ & $\begin{array}{l}0.010^{\star \star} \\
(0.002)\end{array}$ & $\begin{array}{l}0.012^{\star \star} \\
(0.002)\end{array}$ \\
\hline FTHMISS & $\begin{array}{c}0.051^{\star} \\
(0.023)\end{array}$ & $\begin{array}{c}-0.051 \\
(0.036)\end{array}$ & $\begin{array}{c}0.086 \\
(0.045)\end{array}$ & $\begin{array}{c}-0.102 \\
(0.145)\end{array}$ & $\begin{array}{c}-0.010 \\
(0.039)\end{array}$ & $\begin{array}{c}0.107 \\
(0.066)\end{array}$ \\
\hline $\mathrm{INCOME}^{\mathrm{a}}$ & $\begin{array}{l}-0.017^{\star \star} \\
(0.002)\end{array}$ & $\begin{array}{l}-0.011^{\star \star} \\
(0.003)\end{array}$ & $\begin{array}{c}-0.004 \\
(0.003)\end{array}$ & $\begin{array}{c}-0.007 \\
(0.008)\end{array}$ & $\begin{array}{l}-0.008^{\star \star} \\
(0.003)\end{array}$ & $\begin{array}{c}-0.001 \\
(0.001)\end{array}$ \\
\hline BOOKS1 & $\begin{array}{l}-0.113^{\star \star} \\
(0.011)\end{array}$ & $\begin{array}{l}-0.117^{\star \star} \\
(0.018)\end{array}$ & $\begin{array}{l}-0.179^{\star \star} \\
(0.035)\end{array}$ & $\begin{array}{c}-0.135 \\
(0.076)\end{array}$ & $\begin{array}{l}-0.152^{\star \star} \\
(0.022)\end{array}$ & $\begin{array}{c}-0.082 \\
(0.132)\end{array}$ \\
\hline BOOKS3 & $\begin{array}{l}0.086^{\star \star} \\
(0.012)\end{array}$ & $\begin{array}{l}0.098^{\star \star} \\
(0.017)\end{array}$ & $\begin{array}{c}0.048^{\star} \\
(0.022)\end{array}$ & $\begin{array}{c}0.126^{\star} \\
(0.058)\end{array}$ & $\begin{array}{l}0.101^{\star \star} \\
(0.017)\end{array}$ & $\begin{array}{c}0.109^{\star} \\
(0.044)\end{array}$ \\
\hline BOOKS4 & $\begin{array}{l}0.154^{\star \star} \\
(0.014)\end{array}$ & $\begin{array}{l}0.140^{\star \star} \\
(0.018)\end{array}$ & $\begin{array}{l}0.116^{\star \star} \\
(0.021)\end{array}$ & $\begin{array}{c}0.171^{\star} \\
(0.067)\end{array}$ & $\begin{array}{l}0.162^{\star \star} \\
(0.018)\end{array}$ & $\begin{array}{l}0.124^{\star \star} \\
(0.041)\end{array}$ \\
\hline BOOKS5 & $\begin{array}{l}0.209^{\star \star} \\
(0.017)\end{array}$ & $\begin{array}{l}0.231^{\star \star} \\
(0.024)\end{array}$ & $\begin{array}{l}0.141^{\star \star} \\
(0.025)\end{array}$ & $\begin{array}{c}0.072 \\
(0.084)\end{array}$ & $\begin{array}{l}0.184^{\star \star} \\
(0.022)\end{array}$ & $\begin{array}{l}0.141^{\star \star} \\
(0.043)\end{array}$ \\
\hline BOOKS6 & $\begin{array}{l}0.212^{\star \star} \\
(0.019)\end{array}$ & $\begin{array}{l}0.173^{\star \star} \\
(0.028)\end{array}$ & $\begin{array}{l}0.139^{\star \star} \\
(0.027)\end{array}$ & $\begin{array}{l}0.336^{\star \star} \\
(0.093)\end{array}$ & $\begin{array}{l}0.232^{\star \star} \\
(0.023)\end{array}$ & $\begin{array}{l}0.190^{\star \star} \\
(0.042)\end{array}$ \\
\hline BOOKS7 & $\begin{array}{l}0.221^{\star \star} \\
(0.025)\end{array}$ & $\begin{array}{l}0.248^{\star \star} \\
(0.032)\end{array}$ & $\begin{array}{l}0.224^{\star \star} \\
(0.032)\end{array}$ & $\begin{array}{l}0.251^{\star \star} \\
(0.095)\end{array}$ & $\begin{array}{l}0.231^{\star \star} \\
(0.028)\end{array}$ & $\begin{array}{c}0.206^{\star \star} \\
(0.045)\end{array}$ \\
\hline BOOKS8 & $\begin{array}{l}0.285^{\star \star} \\
(0.015)\end{array}$ & $\begin{array}{l}0.288^{\star \star} \\
(0.020)\end{array}$ & $\begin{array}{l}0.223^{\star \star} \\
(0.022)\end{array}$ & $\begin{array}{l}0.244^{\star \star} \\
(0.057)\end{array}$ & $\begin{array}{l}0.290^{\star \star} \\
(0.018)\end{array}$ & $\begin{array}{l}0.254^{\star \star} \\
(0.040)\end{array}$ \\
\hline PCTNATAM & $\begin{array}{l}-0.004^{\star \star} \\
(0.001)\end{array}$ & $\begin{array}{c}-0.0005 \\
(0.002)\end{array}$ & $\begin{array}{c}-0.003 \\
(0.003)\end{array}$ & $\begin{array}{c}-0.002 \\
(0.003)\end{array}$ & $\begin{array}{c}-0.004^{\star \star} \\
(0.001)\end{array}$ & $\begin{array}{c}0.005 \\
(0.005)\end{array}$ \\
\hline AVMTHSCH & $\begin{array}{l}0.107^{\star \star} \\
(0.011)\end{array}$ & $\begin{array}{l}0.108^{\star \star} \\
(0.020)\end{array}$ & $\begin{array}{l}0.089^{\star \star} \\
(0.022)\end{array}$ & $\begin{array}{c}0.099 \\
(0.064)\end{array}$ & $\begin{array}{l}0.123^{\star \star} \\
(0.018)\end{array}$ & $\begin{array}{c}0.048^{\star} \\
(0.023)\end{array}$ \\
\hline AVFTHSCH & $\begin{array}{c}0.019 \\
(0.010)\end{array}$ & $\begin{array}{c}0.003 \\
(0.018)\end{array}$ & $\begin{array}{c}0.045^{\star} \\
(0.023)\end{array}$ & $\begin{array}{c}0.012 \\
(0.064)\end{array}$ & $\begin{array}{c}0.030 \\
(0.018)\end{array}$ & $\begin{array}{c}-0.011 \\
(0.021)\end{array}$ \\
\hline AVINCOME $^{\mathrm{a}}$ & $\begin{array}{c}0.024 \\
(0.031)\end{array}$ & $\begin{array}{l}0.085^{\star \star} \\
(0.029)\end{array}$ & $\begin{array}{c}-0.001 \\
(0.016)\end{array}$ & $\begin{array}{c}-0.015 \\
(0.080)\end{array}$ & $\begin{array}{c}-0.035 \\
(0.021)\end{array}$ & $\begin{array}{c}0.011^{\star} \\
(0.005)\end{array}$ \\
\hline RURAL & $\begin{array}{l}0.080^{\star \star} \\
(0.026)\end{array}$ & $\begin{array}{c}0.032 \\
(0.054)\end{array}$ & $\begin{array}{c}-0.069 \\
(0.088)\end{array}$ & $\begin{array}{c}-0.263^{\star \star} \\
(0.098)\end{array}$ & $\begin{array}{c}-0.002 \\
(0.062)\end{array}$ & $\begin{array}{c}-0.169^{\star} \\
(0.069)\end{array}$ \\
\hline CONSTANT & $\begin{array}{l}-1.863^{\star \star} \\
(0.063)\end{array}$ & $\begin{array}{l}-1.817^{\star \star} \\
(0.106)\end{array}$ & $\begin{array}{l}-1.699 \star \star \\
(0.120)\end{array}$ & $\begin{array}{l}-1.591^{\star \star} \\
(0.279)\end{array}$ & $\begin{array}{l}-1.958^{\star \star} \\
(0.096)\end{array}$ & $\begin{array}{c}-0.562 \\
(0.305)\end{array}$ \\
\hline Number of individuals & 62684 & 30050 & 18442 & 2268 & 31249 & 14179 \\
\hline$R^{2}$ & 0.163 & 0.142 & 0.186 & 0.187 & 0.183 & 0.083 \\
\hline
\end{tabular}

$\star \star$ Statistically significant at $1 \%$, statistically significant at $5 \%$.

Note: Huber-White standard errors, adjusted for the clustering of students within schools, are presented in parentheses. Regional dummy variables were also included in regressions. 
Table 6. Mathematics achievement regressions without selectivity correction

\begin{tabular}{|c|c|c|c|c|c|c|}
\hline & \multicolumn{6}{|c|}{ Dependent variable: MATH } \\
\hline & $\begin{array}{l}\text { Public } \\
\text { DAEM }\end{array}$ & $\begin{array}{c}\text { Public } \\
\text { corporation }\end{array}$ & $\begin{array}{l}\text { Catholic } \\
\text { voucher }\end{array}$ & $\begin{array}{c}\text { Protestant } \\
\text { voucher }\end{array}$ & $\begin{array}{c}\text { Non-religious } \\
\text { voucher }\end{array}$ & $\begin{array}{c}\text { Private } \\
\text { non- } \\
\text { voucher }\end{array}$ \\
\hline FEMALE & $\begin{array}{c}-0.054^{\star} \\
(0.022)\end{array}$ & $\begin{array}{c}-0.045 \\
(0.023)\end{array}$ & $\begin{array}{c}-0.043 \\
(0.024)\end{array}$ & $\begin{array}{c}-0.074 \\
(0.041)\end{array}$ & $\begin{array}{l}-0.049^{\star \star} \\
(0.018)\end{array}$ & $\begin{array}{c}0.047 \\
(0.025)\end{array}$ \\
\hline NATAM & $\begin{array}{l}-0.083^{\star \star} \\
(0.014)\end{array}$ & $\begin{array}{c}0.013 \\
(0.024)\end{array}$ & $\begin{array}{c}-0.064 \\
(0.039)\end{array}$ & $\begin{array}{c}-0.077 \\
(0.068)\end{array}$ & $\begin{array}{c}-0.017 \\
(0.022)\end{array}$ & $\begin{array}{c}-0.067 \\
(0.066)\end{array}$ \\
\hline MTHSCH & $\begin{array}{l}0.016^{\star \star} \\
(0.001)\end{array}$ & $\begin{array}{l}0.014^{\star \star} \\
(0.002)\end{array}$ & $\begin{array}{l}0.013^{\star \star} \\
(0.002)\end{array}$ & $\begin{array}{c}0.018^{\star} \\
(0.007)\end{array}$ & $\begin{array}{l}0.014^{\star \star} \\
(0.002)\end{array}$ & $\begin{array}{l}0.021^{\star \star} \\
(0.003)\end{array}$ \\
\hline MTHMISS & $\begin{array}{c}0.023 \\
(0.029)\end{array}$ & $\begin{array}{c}0.000 \\
(0.041)\end{array}$ & $\begin{array}{c}0.050 \\
(0.062)\end{array}$ & $\begin{array}{c}0.271 \\
(0.168)\end{array}$ & $\begin{array}{c}-0.005 \\
(0.046)\end{array}$ & $\begin{array}{c}0.218^{\star} \\
(0.087)\end{array}$ \\
\hline FTHSCH & $\begin{array}{l}0.007^{\star \star} \\
(0.001)\end{array}$ & $\begin{array}{l}0.005^{\star \star} \\
(0.002)\end{array}$ & $\begin{array}{l}0.008^{\star \star} \\
(0.002)\end{array}$ & $\begin{array}{c}0.009 \\
(0.008)\end{array}$ & $\begin{array}{l}0.007^{\star \star} \\
(0.002)\end{array}$ & $\begin{array}{l}0.012^{\star \star} \\
(0.003)\end{array}$ \\
\hline FTHMISS & $\begin{array}{c}-0.023 \\
(0.022)\end{array}$ & $\begin{array}{c}-0.049 \\
(0.032)\end{array}$ & $\begin{array}{c}0.054 \\
(0.053)\end{array}$ & $\begin{array}{c}-0.070 \\
(0.117)\end{array}$ & $\begin{array}{l}-0.101^{\star \star} \\
(0.036)\end{array}$ & $\begin{array}{c}0.041 \\
(0.075)\end{array}$ \\
\hline $\mathrm{INCOME}^{\mathrm{a}}$ & $\begin{array}{l}-0.016^{\star \star} \\
(0.002)\end{array}$ & $\begin{array}{c}-0.005^{\star} \\
(0.002)\end{array}$ & $\begin{array}{c}0.002 \\
(0.003)\end{array}$ & $\begin{array}{c}-0.006 \\
(0.012)\end{array}$ & $\begin{array}{c}-0.004 \\
(0.002)\end{array}$ & $\begin{array}{c}-0.001 \\
(0.001)\end{array}$ \\
\hline BOOKS1 & $\begin{array}{l}-0.068^{\star \star} \\
(0.012)\end{array}$ & $\begin{array}{l}-0.095^{\star \star} \\
(0.019)\end{array}$ & $\begin{array}{l}-0.121^{\star \star} \\
(0.035)\end{array}$ & $\begin{array}{c}-0.067 \\
(0.074)\end{array}$ & $\begin{array}{l}-0.104^{\star \star} \\
(0.022)\end{array}$ & $\begin{array}{c}-0.095 \\
(0.166)\end{array}$ \\
\hline BOOKS3 & $\begin{array}{l}0.058^{\star \star} \\
(0.012)\end{array}$ & $\begin{array}{l}0.072^{\star \star} \\
(0.016)\end{array}$ & $\begin{array}{c}0.017 \\
(0.022)\end{array}$ & $\begin{array}{c}0.098 \\
(0.062)\end{array}$ & $\begin{array}{l}0.075^{\star \star} \\
(0.017)\end{array}$ & $\begin{array}{c}0.036 \\
(0.044)\end{array}$ \\
\hline BOOKS4 & $\begin{array}{l}0.110^{\star \star} \\
(0.014)\end{array}$ & $\begin{array}{l}0.111^{\star \star} \\
(0.017)\end{array}$ & $\begin{array}{l}0.090^{\star \star} \\
(0.023)\end{array}$ & $\begin{array}{l}0.162^{\star \star} \\
(0.055)\end{array}$ & $\begin{array}{l}0.117^{\star \star} \\
(0.018)\end{array}$ & $\begin{array}{c}0.051 \\
(0.041)\end{array}$ \\
\hline BOOKS5 & $\begin{array}{l}0.161^{\star \star} \\
(0.018)\end{array}$ & $\begin{array}{l}0.152^{\star \star} \\
(0.024)\end{array}$ & $\begin{array}{l}0.108^{\star \star} \\
(0.025)\end{array}$ & $\begin{array}{c}0.124 \\
(0.078)\end{array}$ & $\begin{array}{l}0.172^{\star \star} \\
(0.022)\end{array}$ & $\begin{array}{c}0.072 \\
(0.046)\end{array}$ \\
\hline BOOKS6 & $\begin{array}{l}0.140^{\star \star} \\
(0.020)\end{array}$ & $\begin{array}{l}0.136^{\star \star} \\
(0.026)\end{array}$ & $\begin{array}{l}0.111^{\star \star} \\
(0.031)\end{array}$ & $\begin{array}{l}0.288^{\star \star} \\
(0.081)\end{array}$ & $\begin{array}{l}0.159^{\star \star} \\
(0.024)\end{array}$ & $\begin{array}{c}0.116^{\star} \\
(0.046)\end{array}$ \\
\hline BOOKS7 & $\begin{array}{l}0.164^{\star \star} \\
(0.027)\end{array}$ & $\begin{array}{l}0.136^{\star \star} \\
(0.033)\end{array}$ & $\begin{array}{l}0.188^{\star \star} \\
(0.034)\end{array}$ & $\begin{array}{l}0.269^{\star \star} \\
(0.093)\end{array}$ & $\begin{array}{l}0.213^{\star \star} \\
(0.032)\end{array}$ & $\begin{array}{l}0.145^{\star \star} \\
(0.048)\end{array}$ \\
\hline BOOKS8 & $\begin{array}{l}0.261^{\star \star} \\
(0.017)\end{array}$ & $\begin{array}{l}0.251^{\star \star} \\
(0.019)\end{array}$ & $\begin{array}{l}0.188^{\star \star} \\
(0.022)\end{array}$ & $\begin{array}{l}0.225^{\star \star} \\
(0.050)\end{array}$ & $\begin{array}{l}0.254^{\star \star} \\
(0.019)\end{array}$ & $\begin{array}{l}0.216^{\star \star} \\
(0.041)\end{array}$ \\
\hline PCTNATAM & $\begin{array}{l}-0.003^{\star \star} \\
(0.001)\end{array}$ & $\begin{array}{c}-0.002 \\
(0.002)\end{array}$ & $\begin{array}{l}0.00004 \\
(0.004)\end{array}$ & $\begin{array}{c}-0.003 \\
(0.003)\end{array}$ & $\begin{array}{c}-0.0002 \\
(0.002)\end{array}$ & $\begin{array}{c}0.004 \\
(0.008)\end{array}$ \\
\hline AVMTHSCH & $\begin{array}{l}0.111^{\star \star} \\
(0.014)\end{array}$ & $\begin{array}{l}0.128^{\star \star} \\
(0.025)\end{array}$ & $\begin{array}{l}0.101^{\star \star} \\
(0.031)\end{array}$ & $\begin{array}{c}0.127 \\
(0.064)\end{array}$ & $\begin{array}{l}0.130^{\star \star} \\
(0.023)\end{array}$ & $\begin{array}{c}0.054 \\
(0.030)\end{array}$ \\
\hline AVFTHSCH & $\begin{array}{c}0.009 \\
(0.013)\end{array}$ & $\begin{array}{c}-0.034 \\
(0.024)\end{array}$ & $\begin{array}{c}0.033 \\
(0.030)\end{array}$ & $\begin{array}{c}-0.022 \\
(0.066)\end{array}$ & $\begin{array}{c}0.027 \\
(0.023)\end{array}$ & $\begin{array}{c}-0.030 \\
(0.029)\end{array}$ \\
\hline AVINCOME ${ }^{\mathrm{a}}$ & $\begin{array}{c}0.059 \\
(0.044)\end{array}$ & $\begin{array}{l}0.144^{\star \star} \\
(0.044)\end{array}$ & $\begin{array}{c}0.030 \\
(0.020)\end{array}$ & $\begin{array}{c}-0.075 \\
(0.089)\end{array}$ & $\begin{array}{c}-0.009 \\
(0.026)\end{array}$ & $\begin{array}{l}0.026^{\star \star} \\
(0.008)\end{array}$ \\
\hline RURAL & $\begin{array}{l}0.215^{\star \star} \\
(0.034)\end{array}$ & $\begin{array}{c}0.120^{\star} \\
(0.058)\end{array}$ & $\begin{array}{c}0.046 \\
(0.112)\end{array}$ & $\begin{array}{c}-0.101 \\
(0.082)\end{array}$ & $\begin{array}{c}0.083 \\
(0.119)\end{array}$ & $\begin{array}{c}-0.158 \\
(0.085)\end{array}$ \\
\hline CONSTANT & $\begin{array}{l}-1.655^{\star \star} \\
(0.077)\end{array}$ & $\begin{array}{l}-1.612^{\star \star} \\
(0.145)\end{array}$ & $\begin{array}{l}-1.576^{\star \star} \\
(0.159)\end{array}$ & $\begin{array}{l}-1.369^{\star \star} \\
(0.243)\end{array}$ & $\begin{array}{l}-1.931^{\star \star} \\
(0.129)\end{array}$ & $\begin{array}{c}-0.384 \\
(0.467)\end{array}$ \\
\hline Number of individuals & 62684 & 30050 & 18442 & 2268 & 31249 & 14179 \\
\hline$R^{2}$ & 0.123 & 0.124 & 0.167 & 0.147 & 0.161 & 0.081 \\
\hline
\end{tabular}

$\star \star$ Statistically significant at $1 \%, \star$ statistically significant at $5 \%$.

Note: Huber-White standard errors, adjusted for the clustering of students within schools, are presented in parentheses. Regional dummy variables were also included in regressions. 
Table 7. Differences between school types and public DAEM for student with average characteristics of DAEM student

\begin{tabular}{|c|c|c|c|c|c|}
\hline & $\begin{array}{c}\text { Public } \\
\text { corporation }\end{array}$ & $\begin{array}{l}\text { Catholic } \\
\text { voucher }\end{array}$ & $\begin{array}{c}\text { Protestant } \\
\text { voucher }\end{array}$ & $\begin{array}{l}\text { Non- } \\
\text { religious } \\
\text { voucher }\end{array}$ & $\begin{array}{c}\text { Private } \\
\text { non- } \\
\text { voucher }\end{array}$ \\
\hline \multicolumn{6}{|l|}{ SPANISH } \\
\hline Unadjusted difference & $\begin{array}{c}0.015 \\
(0.029)\end{array}$ & $\begin{array}{c}0.618 \\
(0.030)\end{array}$ & $\begin{array}{c}0.141 \\
(0.064)\end{array}$ & $\begin{array}{c}0.228 \\
(0.028)\end{array}$ & $\begin{array}{c}1.051 \\
(0.025)\end{array}$ \\
\hline \multicolumn{6}{|l|}{ Difference adjusted for: } \\
\hline Individual SES & $\begin{array}{c}-0.085 \\
(0.022)\end{array}$ & $\begin{array}{c}0.315 \\
(0.028)\end{array}$ & $\begin{array}{c}0.023 \\
(0.040)\end{array}$ & $\begin{array}{c}0.029 \\
(0.025)\end{array}$ & $\begin{array}{c}0.619 \\
(0.052)\end{array}$ \\
\hline Individual SES/peer SES & $\begin{array}{l}-0.128 \\
(0.019)\end{array}$ & $\begin{array}{c}0.091 \\
(0.031)\end{array}$ & $\begin{array}{c}-0.096 \\
(0.054)\end{array}$ & $\begin{array}{c}-0.074 \\
(0.024)\end{array}$ & $\begin{array}{c}0.457 \\
(0.128)\end{array}$ \\
\hline Individual SES/peer SES/selectivity & $\begin{array}{c}-0.240 \\
(0.088)\end{array}$ & $\begin{array}{c}-0.055 \\
(0.136)\end{array}$ & $\begin{array}{c}1.309 \\
(0.614)\end{array}$ & $\begin{array}{c}-0.122 \\
(0.108)\end{array}$ & $\begin{array}{c}0.120 \\
(0.163)\end{array}$ \\
\hline \multicolumn{6}{|l|}{ MATH } \\
\hline Unadjusted difference & $\begin{array}{c}-0.079 \\
(0.033)\end{array}$ & $\begin{array}{c}0.561 \\
(0.036)\end{array}$ & $\begin{array}{c}-0.008 \\
(0.059)\end{array}$ & $\begin{array}{c}0.121 \\
(0.034)\end{array}$ & $\begin{array}{c}1.087 \\
(0.031)\end{array}$ \\
\hline \multicolumn{6}{|l|}{ Difference adjusted for: } \\
\hline Individual SES & $\begin{array}{c}-0.164 \\
(0.028)\end{array}$ & $\begin{array}{c}0.352 \\
(0.035)\end{array}$ & $\begin{array}{c}-0.183 \\
(0.040)\end{array}$ & $\begin{array}{c}0.002 \\
(0.031)\end{array}$ & $\begin{array}{c}0.623 \\
(0.074)\end{array}$ \\
\hline Individual SES/peer SES & $\begin{array}{c}-0.213 \\
(0.025)\end{array}$ & $\begin{array}{c}0.120 \\
(0.042)\end{array}$ & $\begin{array}{c}-0.268 \\
(0.047)\end{array}$ & $\begin{array}{c}-0.123 \\
(0.032)\end{array}$ & $\begin{array}{c}0.468 \\
(0.204)\end{array}$ \\
\hline Individual SES/peer SES/selectivity & $\begin{array}{c}-0.285 \\
(0.125)\end{array}$ & $\begin{array}{c}-0.109 \\
(0.191)\end{array}$ & $\begin{array}{c}0.503 \\
(0.548)\end{array}$ & $\begin{array}{c}-0.262 \\
(0.149)\end{array}$ & $\begin{array}{c}0.029 \\
(0.237)\end{array}$ \\
\hline
\end{tabular}

Note: Standard errors are in parentheses.

ordinary least-squares regressions that included school-type dummies as the only regressors). As in prior analyses, students in every type of private school have higher Spanish achievement than DAEM students. For example, private non-voucher students score more than one standard deviation higher than public students. The uncorrected advantage of Catholic students is around 0.6 standard deviations. Raw differences on the mathematics test are similar, except that students in public corporation and Protestant schools have slightly lower achievement than DAEM students.

Now consider the effects of each school type on Spanish and mathematics achievement, correcting for both student and peer variables (these results were calculated with the regression estimates from Tables 5 and 6). Two school types have positive effects on Spanish achievement: Catholic voucher schools (0.09) and private non-voucher (0.46). The effects on mathematics achievement are quite similar. In contrast, the other school types - public corporation, Protestant, and non-religious voucher - all have somewhat lower achievement than DAEM schools. The disadvantage of non-religious voucher students is around 0.07 in Spanish, and 0.12 in mathematics.

Are the magnitudes of these effects noteworthy? Catholic schools have an effect size of around 0.09, squarely in the range of Catholic effects in the US, which some have argued to be of negligible practical import (Witte, 1992; Levin, 1998). ${ }^{17}$ The effect sizes of non-religious voucher schools in Spanish and mathematics, although 
consistently negative, are also fairly small. Finally, private non-voucher schools have quite substantial effect sizes, nearly one-half of a standard deviation.

For purposes of comparison, I estimated the same effects with regressions that include controls for individual SES (socio-economic status), but exclude peer variables. These effects are also reported in Table 7, although the underlying regression estimates are not reported. ${ }^{18}$ Prior studies of private school achievement have acknowledged that omission of peer-group measures could bias private school effects. ${ }^{19}$ If private school students are generally of higher socio-economic status, as in Chile, and such peer-group characteristics are positively related to achievement, then the omission of peer attributes would probably lead to overstatement of private school effects. Indeed, estimated private school effects that are based on the reduced regressions are larger in every category. For example, the Catholic school effect for the Spanish test is over three times the magnitude of the effect that is uncorrected for peer attributes.

I present these results to emphasize an important aspect of private and public comparisons: that poorly measured or omitted peer attributes are likely to severely bias estimates of private school effects. Furthermore, there is good reason to be concerned about measurement error in peer attributes. Most private/public comparisons in the US and developing countries are based on samples of data, rather than a census. Thus, measures of peer attributes are typically based on averages over a sample of students from each school. But two factors are rarely considered: (1) whether school-wide averages yield a statistically precise measure of school peer attributes, and (2) whether the school rather than the classroom is the appropriate unit of analysis for peer interactions. ${ }^{20}$ Either could lead to measurement error in peer variables. One advantage of the quasi-census used in this study is the ability to obtain precise measures of peer characteristics at the classroom level.

Thus far, the analyses have compared achievement in DAEM schools with other school types, given a student with the average characteristics of DAEM students. Table 8 uses a different benchmark, comparing achievement of public corporation schools with other school types, given a student with the average characteristics of corporation students. These effects turn out to be somewhat larger. After adjusting for individual and peer SES, the effects of Catholic schools on Spanish and mathematics achievement are 0.18 and 0.26 , respectively. Likewise, the effects of non-voucher schools increase slightly to 0.48 and 0.53 , respectively. Non-religious voucher schools have rather small positive effects in Spanish and mathematics, although neither effect is more than twice its standard error.

\section{A Model of School Choice}

Table 9 presents a multinomial logit model of choice among the six school categories, estimated with the full sample of 158872 observations. I shall subsequently use model results to assess whether selection bias is present in the previous estimates of private school effects. The model's independent variables include the student and location variables already described, presuming that all are associated with the likelihood of choosing private or public schools. An additional variable, DENSITY, is included in the choice model, but excluded from subsequent achievement regressions. It measures the number of schools of each type per square kilometer in each student's municipality. ${ }^{21}$ Note that DENSITY is choice specific, unlike other independent variables that are individual specific. Therefore, six densities are calculated for each individual in order to gauge the relative supply of 
Table 8. Differences between school types and public corporation for student with average characteristics of corporation student

\begin{tabular}{|c|c|c|c|c|}
\hline & $\begin{array}{l}\text { Catholic } \\
\text { voucher }\end{array}$ & $\begin{array}{c}\text { Protestant } \\
\text { voucher }\end{array}$ & $\begin{array}{c}\text { Non- } \\
\text { religious } \\
\text { voucher }\end{array}$ & $\begin{array}{c}\text { Private } \\
\text { non- } \\
\text { voucher }\end{array}$ \\
\hline \multicolumn{5}{|l|}{ SPANISH } \\
\hline Unadjusted difference & $\begin{array}{c}0.602 \\
(0.032)\end{array}$ & $\begin{array}{c}0.125 \\
(0.064)\end{array}$ & $\begin{array}{c}0.213 \\
(0.030)\end{array}$ & $\begin{array}{c}1.036 \\
(0.027)\end{array}$ \\
\hline \multicolumn{5}{|l|}{ Difference adjusted for: } \\
\hline Individual SES & $\begin{array}{c}0.360 \\
(0.029)\end{array}$ & $\begin{array}{c}0.086 \\
(0.066)\end{array}$ & $\begin{array}{c}0.135 \\
(0.024)\end{array}$ & $\begin{array}{c}0.643 \\
(0.044)\end{array}$ \\
\hline Individual SES/peer SES & $\begin{array}{c}0.180 \\
(0.030)\end{array}$ & $\begin{array}{c}-0.004 \\
(0.071)\end{array}$ & $\begin{array}{c}0.043 \\
(0.024)\end{array}$ & $\begin{array}{c}0.483 \\
(0.105)\end{array}$ \\
\hline Individual SES/peer SES/selectivity & $\begin{array}{c}0.145 \\
(0.133)\end{array}$ & $\begin{array}{c}1.652 \\
(0.673)\end{array}$ & $\begin{array}{c}0.098 \\
(0.090)\end{array}$ & $\begin{array}{c}0.283 \\
(0.139)\end{array}$ \\
\hline \multicolumn{5}{|l|}{ МATH } \\
\hline Unadjusted difference & $\begin{array}{c}0.640 \\
(0.038)\end{array}$ & $\begin{array}{c}0.071 \\
(0.060)\end{array}$ & $\begin{array}{c}0.200 \\
(0.036)\end{array}$ & $\begin{array}{c}1.166 \\
(0.034)\end{array}$ \\
\hline \multicolumn{5}{|l|}{ Difference adjusted for: } \\
\hline Individual SES & $\begin{array}{c}0.454 \\
(0.037)\end{array}$ & $\begin{array}{c}0.009 \\
(0.066)\end{array}$ & $\begin{array}{c}0.135 \\
(0.031)\end{array}$ & $\begin{array}{c}0.700 \\
(0.062)\end{array}$ \\
\hline Individual SES/peer SES & $\begin{array}{c}0.257 \\
(0.041)\end{array}$ & $\begin{array}{c}-0.050 \\
(0.063)\end{array}$ & $\begin{array}{c}0.023 \\
(0.030)\end{array}$ & $\begin{array}{c}0.531 \\
(0.166)\end{array}$ \\
\hline Individual SES/peer SES/selectivity & $\begin{array}{c}0.103 \\
(0.185)\end{array}$ & $\begin{array}{c}0.882 \\
(0.590)\end{array}$ & $\begin{array}{c}-0.035 \\
(0.121)\end{array}$ & $\begin{array}{c}0.203 \\
(0.198)\end{array}$ \\
\hline
\end{tabular}

Note: Standard errors are in parentheses.

each school type available to each student. I hypothesize that the probability of choosing a school alternative is positively related to the relative availability of that alternative in the family's municipality. The analysis treats DENSITY as an exogenous determinant of school choice that is unrelated to student achievement (in the next section, I provide evidence that this assumption is appropriate). ${ }^{22}$

Five coefficients are estimated for each individual-specific variable in the choice model. Each coefficient is interpreted relative to the omitted alternative of public DAEM schools. Thus, the positive and statistically significant coefficient of FEMALE for the Catholic alternative indicates that girls, all else equal, are more likely to attend Catholic schools than the public DAEM alternative. Other coefficients do little to alter impressions left by the simple descriptive statistics reported in Table 4. For example, children are more likely to attend private schools whose parents have higher incomes and greater schooling. The same pattern exists for BOOKS, a proxy of family socio-economic status.

A single coefficient is estimated for each choice-specific variable, including DENSITY and its squared value. ${ }^{23}$ The positive coefficient on DENSITY accords with expectations - that increasing availability of a given school type in a municipality increases the probability that it is chosen, all else equal. The negative coefficient on the squared value of DENSITY indicates that while the probability increases, it does so at a decreasing rate. Both coefficients are statistically significant. 
Table 9. A multinomial logit model of school choice

\begin{tabular}{|c|c|c|c|c|c|}
\hline & $\begin{array}{c}\text { Public } \\
\text { corporation }\end{array}$ & $\begin{array}{l}\text { Catholic } \\
\text { voucher }\end{array}$ & $\begin{array}{c}\text { Protestant } \\
\text { voucher }\end{array}$ & $\begin{array}{l}\text { Non- } \\
\text { religious } \\
\text { voucher }\end{array}$ & $\begin{array}{c}\text { Private } \\
\text { non- } \\
\text { voucher }\end{array}$ \\
\hline \multicolumn{6}{|l|}{ Individual-specific variables } \\
\hline FEMALE & $\begin{array}{c}0.031^{\star} \\
(0.015)\end{array}$ & $\begin{array}{c}0.466^{\star \star} \\
(0.018)\end{array}$ & $\begin{array}{c}0.068 \\
(0.043)\end{array}$ & $\begin{array}{c}-0.025 \\
(0.016)\end{array}$ & $\begin{array}{c}0.037^{\star \star} \\
(0.028)\end{array}$ \\
\hline NATAM & $\begin{array}{l}-0.251^{\star \star} \\
(0.035)\end{array}$ & $\begin{array}{l}-0.307^{\star \star} \\
(0.051)\end{array}$ & $\begin{array}{c}0.163 \\
(0.083)\end{array}$ & $\begin{array}{l}0.161^{\star \star} \\
(0.036)\end{array}$ & $\begin{array}{l}-1.139^{\star \star} \\
(0.136)\end{array}$ \\
\hline MTHSCH & $\begin{array}{l}-0.006 \\
(0.003)\end{array}$ & $\begin{array}{l}0.102^{\star \star} \\
(0.004)\end{array}$ & $\begin{array}{l}0.059^{\star \star} \\
(0.008)\end{array}$ & $\begin{array}{l}0.048^{\star \star} \\
(0.003)\end{array}$ & $\begin{array}{l}0.203^{\star \star} \\
(0.006)\end{array}$ \\
\hline MTHMISS & $\begin{array}{l}-0.060 \\
(0.064)\end{array}$ & $\begin{array}{l}0.789^{\star \star} \\
(0.086)\end{array}$ & $\begin{array}{l}0.616^{\star \star} \\
(0.183)\end{array}$ & $\begin{array}{l}0.320^{\star \star} \\
(0.068)\end{array}$ & $\begin{array}{l}2.257^{\star \star} \\
(0.145)\end{array}$ \\
\hline FTHSCH & $\begin{array}{l}0.024^{\star \star} \\
(0.003)\end{array}$ & $\begin{array}{l}0.069^{\star \star} \\
(0.003)\end{array}$ & $\begin{array}{l}0.071^{\star \star} \\
(0.008)\end{array}$ & $\begin{array}{l}0.042^{\star \star} \\
(0.003)\end{array}$ & $\begin{array}{l}0.166^{\star \star} \\
(0.006)\end{array}$ \\
\hline FTHMISS & $\begin{array}{l}0.162^{\star \star} \\
(0.051)\end{array}$ & $\begin{array}{l}0.490^{\star \star} \\
(0.069)\end{array}$ & $\begin{array}{l}0.757^{\star \star} \\
(0.150)\end{array}$ & $\begin{array}{l}0.309^{\star \star} \\
(0.055)\end{array}$ & $\begin{array}{l}1.950^{\star \star} \\
(0.126)\end{array}$ \\
\hline INCOME & $\begin{array}{l}0.059^{\star \star} \\
(0.005)\end{array}$ & $\begin{array}{l}0.126^{\star \star} \\
(0.005)\end{array}$ & $\begin{array}{l}0.069^{\star \star} \\
(0.012)\end{array}$ & $\begin{array}{l}0.114^{\star \star} \\
(0.005)\end{array}$ & $\begin{array}{l}0.356^{\star \star} \\
(0.005)\end{array}$ \\
\hline BOOKS1 & $\begin{array}{l}-0.193^{\star \star} \\
(0.025)\end{array}$ & $\begin{array}{l}-0.450^{\star \star} \\
(0.041)\end{array}$ & $\begin{array}{l}-0.393^{\star \star} \\
(0.082)\end{array}$ & $\begin{array}{l}-0.285^{\star \star} \\
(0.030)\end{array}$ & $\begin{array}{l}-0.914^{\star \star} \\
(0.158)\end{array}$ \\
\hline BOOKS3 & $\begin{array}{l}0.199^{\star \star} \\
(0.024)\end{array}$ & $\begin{array}{l}0.435^{\star \star} \\
(0.030)\end{array}$ & $\begin{array}{l}0.301^{\star \star} \\
(0.068)\end{array}$ & $\begin{array}{l}0.259^{\star \star} \\
(0.025)\end{array}$ & $\begin{array}{c}0.680^{\star \star} \\
(0.070)\end{array}$ \\
\hline BOOKS4 & $\begin{array}{l}0.278^{\star \star} \\
(0.027)\end{array}$ & $\begin{array}{l}0.571^{\star \star} \\
(0.032)\end{array}$ & $\begin{array}{l}0.371^{\star \star} \\
(0.075)\end{array}$ & $\begin{array}{l}0.408^{\star \star} \\
(0.027)\end{array}$ & $\begin{array}{l}1.152^{\star \star} \\
(0.066)\end{array}$ \\
\hline BOOKS5 & $\begin{array}{l}0.342^{\star \star} \\
(0.035)\end{array}$ & $\begin{array}{l}0.751^{\star \star} \\
(0.038)\end{array}$ & $\begin{array}{l}0.540^{\star \star} \\
(0.092)\end{array}$ & $\begin{array}{l}0.525^{\star \star} \\
(0.034)\end{array}$ & $\begin{array}{l}1.255^{\star \star} \\
(0.072)\end{array}$ \\
\hline BOOKS6 & $\begin{array}{l}0.374^{\star \star} \\
(0.039)\end{array}$ & $\begin{array}{c}0.757^{\star \star} \\
(0.042)\end{array}$ & $\begin{array}{l}0.321^{\star \star} \\
(0.112)\end{array}$ & $\begin{array}{l}0.506^{\star \star} \\
(0.038)\end{array}$ & $\begin{array}{l}1.342^{\star \star} \\
(0.073)\end{array}$ \\
\hline BOOKS7 & $\begin{array}{l}0.324^{\star \star} \\
(0.049)\end{array}$ & $\begin{array}{l}0.842^{\star \star} \\
(0.050)\end{array}$ & $\begin{array}{l}0.634^{\star \star} \\
(0.123)\end{array}$ & $\begin{array}{l}0.519^{\star \star} \\
(0.046)\end{array}$ & $\begin{array}{l}1.478^{\star \star} \\
(0.081)\end{array}$ \\
\hline BOOKS8 & $\begin{array}{l}0.298^{\star \star} \\
(0.027)\end{array}$ & $\begin{array}{l}0.742^{\star \star} \\
(0.030)\end{array}$ & $\begin{array}{l}0.504^{\star \star} \\
(0.072)\end{array}$ & $\begin{array}{l}0.521^{\star \star} \\
(0.026)\end{array}$ & $\begin{array}{l}1.445^{\star \star} \\
(0.060)\end{array}$ \\
\hline RURAL & $\begin{array}{l}-0.425^{\star \star} \\
(0.025)\end{array}$ & $\begin{array}{l}-1.415^{\star \star} \\
(0.047)\end{array}$ & $\begin{array}{l}0.726^{\star \star} \\
(0.060)\end{array}$ & $\begin{array}{l}-0.742^{\star \star} \\
(0.033)\end{array}$ & $\begin{array}{l}-1.541^{\star \star} \\
(0.113)\end{array}$ \\
\hline CONSTANT & $\begin{array}{l}-1.231^{\star \star} \\
(0.029)\end{array}$ & $\begin{array}{l}-2.821^{\star \star} \\
(0.041)\end{array}$ & $\begin{array}{l}-5.010^{\star \star} \\
(0.107)\end{array}$ & $\begin{array}{l}-1.792^{\star \star} \\
(0.032)\end{array}$ & $\begin{array}{l}-7.944^{\star \star} \\
(0.089)\end{array}$ \\
\hline \multicolumn{6}{|l|}{ Choice-specific variables } \\
\hline DENSITY & & & $\begin{array}{l}4.216^{\star \star} \\
(0.030)\end{array}$ & & \\
\hline DENSITY $^{2}$ & & & $\begin{array}{l}-1.359^{\star \star} \\
(0.014)\end{array}$ & & \\
\hline Number of observations & & & 158872 & & \\
\hline Log likelihood & & & -179043 & & \\
\hline
\end{tabular}

$\star \star$ Statistically significant at $1 \%,{ }^{\star}$ Statistically significant at $5 \%$.

Note: The coefficients for individual-specific variables are interpreted relative to Public DAEM schools. Regional dummy variables were also included in regressions. 


\section{The Validity of Exclusion Restrictions}

The credibility of the empirical strategy to correct for selection bias hinges on whether the models are correctly specified. In particular, I assume that private school selection is strongly influenced by local school supply (proxied by school densities), but that school densities do not influence achievement. The previous section demonstrated that the first assumption is tenable. However, there are reasons to suspect that the second assumption is less reasonable. For example, private schools may be inclined to locate in communities with high-SES families (or other variables that positively influence achievement). If these variables are not perfectly controlled for in achievement regressions, then private school densities may partially reflect unobserved characteristics of families or communities. Thus, densities cannot properly be excluded from achievement regressions.

As a straightforward test, I re-estimated the six achievement regressions from Tables 5 and 6 , including the density measures as additional independent variables. I interpret statistically significant correlations between the density variables and achievement-conditional on observed student and peer characteristics-as evidence that the exclusion restrictions are not justified. Neal (1997) and Evans and Schwab (1995) pursued a similar approach in their analyses of public and private achievement in the US. As these authors note, this is not a formal specification test, although it does provide suggestive evidence on the validity of the exclusion restrictions.

Tables 10 and 11 present some results from these regressions. Recall that the density variables used in the multinomial logit are choice specific, rather than individual specific, implying six separate densities for each individual. Therefore, the achievement regressions include six density variables, corresponding to each school type, as well as the corresponding squared terms. In the vast majority of cases, these variables are not significantly associated with achievement, after student and peer characteristics are controlled for (only three of 72 density coefficients are statistically significant for SPANISH, with similar results for MATH). I further tested the hypothesis that the coefficients on DENSITY - and its squared termsare jointly equal to zero in the regressions for each school type. In almost every case, I was unable to reject this hypothesis, suggesting that the exclusion restrictions are reasonable. A notable exception is the case of private non-voucher schools and SPANISH (see Table 10), for which I can reject the hypotheses that the DENSITY and DENSITY ${ }^{2}$ coefficients are jointly equal to zero. The results are suggestive that the strategy of correcting for selection bias is less appropriate for comparisons of public and non-voucher private schools.

\section{Correcting for Selection Bias}

Results from the multinomial logit were used to predict $P_{i j}$, the probability that the $i$ th student attends the $j$ th school type. $P_{i j}$ was then used to calculate the selectivity variable, $\lambda_{i j}$. Tables 12 and 13 present estimates of regressions for SPANISH and MATH, respectively, which include $\lambda_{i j}$ as a regressor. One immediate observation is that many coefficients are estimated less precisely than in regressions that exclude the selectivity term. Increased standard errors of coefficients are often a byproduct of two-step corrections, stemming from multicollinearity introduced by the selectivity term; this is especially the case where only a few variables identifying selection are excluded from the principal regressions (Vella, 1998). 
Table 10. Spanish achievement regressions with school densities

\begin{tabular}{|c|c|c|c|c|c|c|}
\hline & \multicolumn{6}{|c|}{ Dependent variable: SPANISH } \\
\hline & $\begin{array}{l}\text { Public } \\
\text { DAEM }\end{array}$ & $\begin{array}{c}\text { Public } \\
\text { corporation }\end{array}$ & $\begin{array}{l}\text { Catholic } \\
\text { voucher }\end{array}$ & $\begin{array}{l}\text { Protestant } \\
\text { voucher }\end{array}$ & $\begin{array}{l}\text { Non- } \\
\text { religious } \\
\text { voucher }\end{array}$ & $\begin{array}{l}\text { Private } \\
\text { non- } \\
\text { voucher }\end{array}$ \\
\hline DENSITY (DAEM) & $\begin{array}{c}-0.119 \\
(0.298)\end{array}$ & $\begin{array}{c}-2.506 \\
(6.227)\end{array}$ & $\begin{array}{c}-0.031 \\
(0.388)\end{array}$ & $\begin{array}{c}1.890 \\
(4.903)\end{array}$ & $\begin{array}{c}-0.058 \\
(0.295)\end{array}$ & $\begin{array}{c}0.592 \\
(0.527)\end{array}$ \\
\hline DENSITY (Corp) & $\begin{array}{c}-0.320 \\
(0.846)\end{array}$ & $\begin{array}{c}0.557 \\
(0.395)\end{array}$ & $\begin{array}{c}-0.047 \\
(0.338)\end{array}$ & $\begin{array}{c}4.968 \\
(5.052)\end{array}$ & $\begin{array}{c}0.149 \\
(0.327)\end{array}$ & $\begin{array}{c}-0.161 \\
(0.516)\end{array}$ \\
\hline DENSITY (Cath) & $\begin{array}{c}-0.788 \\
(0.895)\end{array}$ & $\begin{array}{c}-0.238 \\
(1.000)\end{array}$ & $\begin{array}{c}0.802 \\
(0.951)\end{array}$ & $\begin{array}{c}-8.093^{\star} \\
(4.021)\end{array}$ & $\begin{array}{c}-0.486 \\
(0.611)\end{array}$ & $\begin{array}{c}0.737 \\
(0.933)\end{array}$ \\
\hline DENSITY (Prot) & $\begin{array}{c}-2.982 \\
(3.310)\end{array}$ & $\begin{array}{c}0.422 \\
(4.387)\end{array}$ & $\begin{array}{c}-0.992 \\
(5.509)\end{array}$ & $\begin{array}{c}-24.298 \\
(45.402)\end{array}$ & $\begin{array}{c}2.544 \\
(2.209)\end{array}$ & $\begin{array}{c}5.482 \\
(6.501)\end{array}$ \\
\hline DENSITY (Non-R) & $\begin{array}{c}-0.034 \\
(0.288)\end{array}$ & $\begin{array}{c}-0.379 \\
(0.303)\end{array}$ & $\begin{array}{c}-0.020 \\
(0.233)\end{array}$ & $\begin{array}{c}-0.179 \\
(3.087)\end{array}$ & $\begin{array}{c}-0.148 \\
(0.187)\end{array}$ & $\begin{array}{c}-0.154 \\
(0.246)\end{array}$ \\
\hline DENSITY (Non-V) & $\begin{array}{c}-0.051 \\
(0.601)\end{array}$ & $\begin{array}{c}-0.183 \\
(0.199)\end{array}$ & $\begin{array}{c}0.023 \\
(0.245)\end{array}$ & $\begin{array}{c}-3.355 \\
(10.603)\end{array}$ & $\begin{array}{c}-0.085 \\
(0.224)\end{array}$ & $\begin{array}{c}-0.391^{\star \star} \\
(0.141)\end{array}$ \\
\hline DENSITY $^{2}$ (DAEM) & $\begin{array}{c}0.086 \\
(0.130)\end{array}$ & $\begin{array}{c}39.469 \\
(75.288)\end{array}$ & $\begin{array}{c}-0.030 \\
(0.179)\end{array}$ & $\begin{array}{c}-2.075 \\
(6.165)\end{array}$ & $\begin{array}{c}0.144 \\
(0.142)\end{array}$ & $\begin{array}{c}-0.441 \\
(0.293)\end{array}$ \\
\hline DENSITY $^{2}$ (Corp) & $\begin{array}{c}0.292 \\
(0.573)\end{array}$ & $\begin{array}{c}-0.186 \\
(0.141)\end{array}$ & $\begin{array}{c}-0.007 \\
(0.159)\end{array}$ & $\begin{array}{l}-5.160 \\
(9.378)\end{array}$ & $\begin{array}{c}-0.035 \\
(0.144)\end{array}$ & $\begin{array}{c}0.194 \\
(0.328)\end{array}$ \\
\hline DENSITY $^{2}$ (Cath) & $\begin{array}{c}1.398 \\
(1.395)\end{array}$ & $\begin{array}{c}-2.083 \\
(3.000)\end{array}$ & $\begin{array}{c}-1.384 \\
(1.300)\end{array}$ & $\begin{array}{c}5.767 \\
(46.614)\end{array}$ & $\begin{array}{c}0.645 \\
(0.929)\end{array}$ & $\begin{array}{c}0.243 \\
(1.261)\end{array}$ \\
\hline DENSITY $^{2}$ (Prot) & $\begin{array}{c}29.846 \\
(27.803)\end{array}$ & $\begin{array}{l}-16.683 \\
(31.432)\end{array}$ & $\begin{array}{c}26.821 \\
(48.627)\end{array}$ & $\begin{array}{c}414.188 \\
(1063.592)\end{array}$ & $\begin{array}{c}-18.034 \\
(18.425)\end{array}$ & $\begin{array}{l}-86.670 \\
(65.342)\end{array}$ \\
\hline DENSITY $^{2}$ (Non-R) & $\begin{array}{c}0.044 \\
(0.110)\end{array}$ & $\begin{array}{c}0.094 \\
(0.110)\end{array}$ & $\begin{array}{c}0.010 \\
(0.093)\end{array}$ & $\begin{array}{c}0.279 \\
(3.344)\end{array}$ & $\begin{array}{c}0.055 \\
(0.070)\end{array}$ & $\begin{array}{c}0.040 \\
(0.094)\end{array}$ \\
\hline DENSITY $^{2}$ (Non-V) & $\begin{array}{c}0.758 \\
(0.908)\end{array}$ & $\begin{array}{c}0.108 \\
(0.101)\end{array}$ & $\begin{array}{c}-0.052 \\
(0.124)\end{array}$ & $\begin{array}{c}8.590 \\
(43.020)\end{array}$ & $\begin{array}{c}-0.015 \\
(0.130)\end{array}$ & $\begin{array}{c}0.158^{\star} \\
(0.061)\end{array}$ \\
\hline $\begin{array}{l}p \text { Value of Wald test } \\
\text { that DENSITY } \\
\text { coefficients are } \\
\text { jointly equal to } 0\end{array}$ & 0.34 & 0.28 & 0.92 & 0.11 & 0.77 & 0.04 \\
\hline $\begin{array}{l}p \text { Value of Wald test } \\
\text { that DENSITY } \\
\text { coefficients are } \\
\text { jointly equal to } 0\end{array}$ & 0.06 & 0.32 & 0.68 & 0.01 & 0.72 & 0.03 \\
\hline
\end{tabular}

\footnotetext{
$\star \star$ Statistically significant at $1 \%,{ }^{\star}$ statistically significant at $5 \%$.
}

Note: Huber-White standard errors, adjusted for the clustering of students within schools, are presented in parentheses. All regressions include a constant and the variables in Tables 5 and 6 . 
Table 11. Mathematics achievement regressions with school densities

\begin{tabular}{|c|c|c|c|c|c|c|}
\hline & \multicolumn{6}{|c|}{ Dependent variable: MATH } \\
\hline & $\begin{array}{l}\text { Public } \\
\text { DAEM }\end{array}$ & $\begin{array}{c}\text { Public } \\
\text { corporation }\end{array}$ & $\begin{array}{l}\text { Catholic } \\
\text { voucher }\end{array}$ & $\begin{array}{c}\text { Protestant } \\
\text { voucher }\end{array}$ & $\begin{array}{l}\text { Non- } \\
\text { religious } \\
\text { voucher }\end{array}$ & $\begin{array}{l}\text { Private } \\
\text { non- } \\
\text { voucher }\end{array}$ \\
\hline DENSITY (DAEM) & $\begin{array}{c}0.038 \\
(0.432)\end{array}$ & $\begin{array}{l}-3.510 \\
(8.905)\end{array}$ & $\begin{array}{c}-0.005 \\
(0.405)\end{array}$ & $\begin{array}{c}0.542 \\
(5.159)\end{array}$ & $\begin{array}{c}-0.063 \\
(0.431)\end{array}$ & $\begin{array}{c}0.790 \\
(0.843)\end{array}$ \\
\hline DENSITY (Corp) & $\begin{array}{c}1.183 \\
(0.944)\end{array}$ & $\begin{array}{c}0.747 \\
(0.590)\end{array}$ & $\begin{array}{c}-0.298 \\
(0.394)\end{array}$ & $\begin{array}{c}0.906 \\
(5.244)\end{array}$ & $\begin{array}{c}0.348 \\
(0.418)\end{array}$ & $\begin{array}{c}-0.121 \\
(0.791)\end{array}$ \\
\hline DENSITY (Cath) & $\begin{array}{c}-0.833 \\
(1.385)\end{array}$ & $\begin{array}{c}1.140 \\
(1.339)\end{array}$ & $\begin{array}{c}0.522 \\
(1.040)\end{array}$ & $\begin{array}{c}-3.454 \\
(4.500)\end{array}$ & $\begin{array}{c}-0.481 \\
(0.838)\end{array}$ & $\begin{array}{c}0.807 \\
(1.310)\end{array}$ \\
\hline DENSITY (Prot) & $\begin{array}{c}-4.275 \\
(4.511)\end{array}$ & $\begin{array}{c}5.111 \\
(5.752)\end{array}$ & $\begin{array}{c}3.784 \\
(5.634)\end{array}$ & $\begin{array}{l}-11.399 \\
(52.072)\end{array}$ & $\begin{array}{c}2.164 \\
(3.004)\end{array}$ & $\begin{array}{c}8.693 \\
(10.483)\end{array}$ \\
\hline DENSITY (Non-R) & $\begin{array}{c}0.121 \\
(0.373)\end{array}$ & $\begin{array}{c}-0.976^{\star} \\
(0.449)\end{array}$ & $\begin{array}{c}0.168 \\
(0.299)\end{array}$ & $\begin{array}{c}0.562 \\
(2.695)\end{array}$ & $\begin{array}{c}-0.137 \\
(0.269)\end{array}$ & $\begin{array}{c}-0.511 \\
(0.411)\end{array}$ \\
\hline DENSITY (Non-V) & $\begin{array}{c}-1.685 \\
(1.000)\end{array}$ & $\begin{array}{c}0.020 \\
(0.314)\end{array}$ & $\begin{array}{c}0.283 \\
(0.289)\end{array}$ & $\begin{array}{c}-5.555 \\
(9.596)\end{array}$ & $\begin{array}{c}-0.263 \\
(0.297)\end{array}$ & $\begin{array}{c}-0.521^{\star} \\
(0.207)\end{array}$ \\
\hline DENSITY $^{2}$ (DAEM) & $\begin{array}{c}-0.045 \\
(0.157)\end{array}$ & $\begin{array}{c}58.079 \\
(108.631)\end{array}$ & $\begin{array}{c}-0.028 \\
(0.186)\end{array}$ & $\begin{array}{c}-3.346 \\
(5.478)\end{array}$ & $\begin{array}{c}0.185 \\
(0.212)\end{array}$ & $\begin{array}{c}-0.500 \\
(0.481)\end{array}$ \\
\hline DENSITY $^{2}$ (Corp) & $\begin{array}{c}-0.783 \\
(0.643)\end{array}$ & $\begin{array}{c}-0.166 \\
(0.208)\end{array}$ & $\begin{array}{c}0.113 \\
(0.177)\end{array}$ & $\begin{array}{c}-5.631 \\
(8.123)\end{array}$ & $\begin{array}{c}-0.151 \\
(0.175)\end{array}$ & $\begin{array}{c}0.389 \\
(0.505)\end{array}$ \\
\hline DENSITY $^{2}$ (Cath) & $\begin{array}{c}1.784 \\
(2.292)\end{array}$ & $\begin{array}{c}-6.651 \\
(4.233)\end{array}$ & $\begin{array}{c}-1.356 \\
(1.418)\end{array}$ & $\begin{array}{c}-24.662 \\
(39.215)\end{array}$ & $\begin{array}{c}0.843 \\
(1.270)\end{array}$ & $\begin{array}{c}0.369 \\
(1.837)\end{array}$ \\
\hline DENSITY $^{2}$ (Prot) & $\begin{array}{c}52.369 \\
(36.453)\end{array}$ & $\begin{array}{c}-47.938 \\
(43.449)\end{array}$ & $\begin{array}{l}-39.069 \\
(50.522)\end{array}$ & $\begin{array}{c}653.933 \\
(938.450)\end{array}$ & $\begin{array}{c}-15.014 \\
(24.337)\end{array}$ & $\begin{array}{c}-111.654 \\
(105.224)\end{array}$ \\
\hline DENSITY $^{2}$ (Non-R) & $\begin{array}{c}0.022 \\
(0.140)\end{array}$ & $\begin{array}{c}0.278 \\
(0.152)\end{array}$ & $\begin{array}{c}-0.048 \\
(0.116)\end{array}$ & $\begin{array}{c}0.897 \\
(2.777)\end{array}$ & $\begin{array}{c}0.030 \\
(0.103)\end{array}$ & $\begin{array}{c}0.131 \\
(0.154)\end{array}$ \\
\hline DENSITY $^{2}$ (Non-V) & $\begin{array}{c}2.843^{\star} \\
(1.442)\end{array}$ & $\begin{array}{c}0.040 \\
(0.170)\end{array}$ & $\begin{array}{c}-0.159 \\
(0.147)\end{array}$ & $\begin{array}{c}26.475 \\
(36.028)\end{array}$ & $\begin{array}{c}0.069 \\
(0.170)\end{array}$ & $\begin{array}{c}0.190^{\star} \\
(0.086)\end{array}$ \\
\hline $\begin{array}{l}p \text { Value of Wald test } \\
\text { that DENSITY } \\
\text { coefficients are } \\
\text { jointly equal to } 0\end{array}$ & 0.12 & 0.04 & 0.70 & 0.43 & 0.85 & 0.12 \\
\hline $\begin{array}{l}p \text { Value of Wald test } \\
\text { that DENSITY } \\
\text { coefficients are } \\
\text { jointly equal to } 0\end{array}$ & 0.01 & 0.18 & 0.68 & 0.02 & 0.76 & 0.10 \\
\hline
\end{tabular}

$\star \star$ Statistically significant at $1 \%, \star$ statistically significant at $5 \%$.

Note: Huber-White standard errors, adjusted for the clustering of students within schools, are presented in parentheses. All regressions include a constant and the variables in Tables 5 and 6. 
Table 12. Spanish achievement regressions with selectivity correction

\begin{tabular}{|c|c|c|c|c|c|c|}
\hline & \multicolumn{6}{|c|}{ Dependent variable: SPANISH } \\
\hline & $\begin{array}{l}\text { Public } \\
\text { DAEM }\end{array}$ & $\begin{array}{c}\text { Public } \\
\text { corporation }\end{array}$ & $\begin{array}{l}\text { Catholic } \\
\text { voucher }\end{array}$ & $\begin{array}{l}\text { Protestant } \\
\text { voucher }\end{array}$ & $\begin{array}{l}\text { Non- } \\
\text { religious } \\
\text { voucher }\end{array}$ & $\begin{array}{c}\text { Private } \\
\text { non- } \\
\text { voucher }\end{array}$ \\
\hline FEMALE & $\begin{array}{l}0.203^{\star \star} \\
(0.015)\end{array}$ & $\begin{array}{l}0.189^{\star \star} \\
(0.016)\end{array}$ & $\begin{array}{l}0.211^{\star \star} \\
(0.023)\end{array}$ & $\begin{array}{l}0.184^{\star \star} \\
(0.049)\end{array}$ & $\begin{array}{l}0.182^{\star \star} \\
(0.015)\end{array}$ & $\begin{array}{l}0.217^{\star \star} \\
(0.018)\end{array}$ \\
\hline NATAM & $\begin{array}{l}-0.121^{\star \star} \\
(0.016)\end{array}$ & $\begin{array}{c}-0.044 \\
(0.027)\end{array}$ & $\begin{array}{c}-0.088^{\star} \\
(0.042)\end{array}$ & $\begin{array}{c}-0.077 \\
(0.071)\end{array}$ & $\begin{array}{c}-0.023 \\
(0.026)\end{array}$ & $\begin{array}{c}-0.098 \\
(0.083)\end{array}$ \\
\hline MTHSCH & $\begin{array}{l}0.024^{\star \star} \\
(0.002)\end{array}$ & $\begin{array}{l}0.020^{\star \star} \\
(0.002)\end{array}$ & $\begin{array}{l}0.019^{\star \star} \\
(0.003)\end{array}$ & $\begin{array}{c}0.013 \\
(0.009)\end{array}$ & $\begin{array}{l}0.021^{\star \star} \\
(0.002)\end{array}$ & $\begin{array}{l}0.020^{\star \star} \\
(0.003)\end{array}$ \\
\hline MTHMISS & $\begin{array}{c}0.068^{\star} \\
(0.030)\end{array}$ & $\begin{array}{c}0.073 \\
(0.046)\end{array}$ & $\begin{array}{c}0.084 \\
(0.063)\end{array}$ & $\begin{array}{c}0.186 \\
(0.203)\end{array}$ & $\begin{array}{c}0.057 \\
(0.046)\end{array}$ & $\begin{array}{l}0.209^{\star \star} \\
(0.078)\end{array}$ \\
\hline FTHSCH & $\begin{array}{l}0.014^{\star \star} \\
(0.002)\end{array}$ & $\begin{array}{l}0.010^{\star \star} \\
(0.002)\end{array}$ & $\begin{array}{l}0.014^{\star \star} \\
(0.003)\end{array}$ & $\begin{array}{c}-0.002 \\
(0.009)\end{array}$ & $\begin{array}{l}0.010^{\star \star} \\
(0.002)\end{array}$ & $\begin{array}{l}0.016^{\star \star} \\
(0.003)\end{array}$ \\
\hline FTHMISS & $\begin{array}{l}0.066^{\star \star} \\
(0.024)\end{array}$ & $\begin{array}{c}-0.052 \\
(0.036)\end{array}$ & $\begin{array}{c}0.091 \\
(0.047)\end{array}$ & $\begin{array}{c}-0.226 \\
(0.154)\end{array}$ & $\begin{array}{c}-0.010 \\
(0.039)\end{array}$ & $\begin{array}{c}0.148^{\star} \\
(0.070)\end{array}$ \\
\hline INCOME & $\begin{array}{c}-0.009 \\
(0.006)\end{array}$ & $\begin{array}{l}-0.012^{\star \star} \\
(0.004)\end{array}$ & $\begin{array}{c}-0.004 \\
(0.003)\end{array}$ & $\begin{array}{c}0.006 \\
(0.009)\end{array}$ & $\begin{array}{l}-0.008^{\star \star} \\
(0.003)\end{array}$ & $\begin{array}{c}0.004 \\
(0.002)\end{array}$ \\
\hline BOOKS1 & $\begin{array}{l}-0.121^{\star \star} \\
(0.011)\end{array}$ & $\begin{array}{l}-0.118^{\star \star} \\
(0.018)\end{array}$ & $\begin{array}{l}-0.185^{\star \star} \\
(0.036)\end{array}$ & $\begin{array}{c}-0.077 \\
(0.075)\end{array}$ & $\begin{array}{l}-0.150^{\star \star} \\
(0.022)\end{array}$ & $\begin{array}{c}-0.107 \\
(0.132)\end{array}$ \\
\hline BOOKS3 & $\begin{array}{l}0.095^{\star \star} \\
(0.013)\end{array}$ & $\begin{array}{l}0.098^{\star \star} \\
(0.017)\end{array}$ & $\begin{array}{c}0.054^{\star} \\
(0.025)\end{array}$ & $\begin{array}{c}0.084 \\
(0.061)\end{array}$ & $\begin{array}{l}0.100^{\star \star} \\
(0.018)\end{array}$ & $\begin{array}{l}0.125^{\star \star} \\
(0.045)\end{array}$ \\
\hline BOOKS4 & $\begin{array}{l}0.170^{\star \star} \\
(0.018)\end{array}$ & $\begin{array}{l}0.140^{\star \star} \\
(0.018)\end{array}$ & $\begin{array}{l}0.123^{\star \star} \\
(0.024)\end{array}$ & $\begin{array}{c}0.131 \\
(0.068)\end{array}$ & $\begin{array}{l}0.160^{\star \star} \\
(0.019)\end{array}$ & $\begin{array}{l}0.151^{\star \star} \\
(0.043)\end{array}$ \\
\hline BOOKS5 & $\begin{array}{l}0.230^{\star \star} \\
(0.023)\end{array}$ & $\begin{array}{l}0.231^{\star \star} \\
(0.024)\end{array}$ & $\begin{array}{l}0.149^{\star \star} \\
(0.031)\end{array}$ & $\begin{array}{c}0.018 \\
(0.088)\end{array}$ & $\begin{array}{l}0.182^{\star \star} \\
(0.023)\end{array}$ & $\begin{array}{l}0.172^{\star \star} \\
(0.045)\end{array}$ \\
\hline BOOKS6 & $\begin{array}{l}0.233^{\star \star} \\
(0.025)\end{array}$ & $\begin{array}{l}0.173^{\star \star} \\
(0.028)\end{array}$ & $\begin{array}{l}0.148^{\star \star} \\
(0.031)\end{array}$ & $\begin{array}{c}0.334^{\star \star} \\
(0.095)\end{array}$ & $\begin{array}{l}0.230^{\star \star} \\
(0.024)\end{array}$ & $\begin{array}{l}0.223^{\star \star} \\
(0.044)\end{array}$ \\
\hline BOOKS7 & $\begin{array}{l}0.244^{\star \star} \\
(0.031)\end{array}$ & $\begin{array}{l}0.247^{\star \star} \\
(0.032)\end{array}$ & $\begin{array}{l}0.233^{\star \star} \\
(0.037)\end{array}$ & $\begin{array}{c}0.184 \\
(0.101)\end{array}$ & $\begin{array}{l}0.229^{\star \star} \\
(0.029)\end{array}$ & $\begin{array}{l}0.239^{\star \star} \\
(0.047)\end{array}$ \\
\hline BOOKS8 & $\begin{array}{l}0.309^{\star \star} \\
(0.025)\end{array}$ & $\begin{array}{l}0.287^{\star \star} \\
(0.020)\end{array}$ & $\begin{array}{l}0.231^{\star \star} \\
(0.027)\end{array}$ & $\begin{array}{l}0.201^{\star \star} \\
(0.059)\end{array}$ & $\begin{array}{l}0.288^{\star \star} \\
(0.018)\end{array}$ & $\begin{array}{l}0.287^{\star \star} \\
(0.042)\end{array}$ \\
\hline PCTNATAM & $\begin{array}{l}-0.004^{\star \star} \\
(0.001)\end{array}$ & $\begin{array}{c}-0.0004 \\
(0.002)\end{array}$ & $\begin{array}{c}-0.004 \\
(0.003)\end{array}$ & $\begin{array}{c}-0.001 \\
(0.003)\end{array}$ & $\begin{array}{l}-0.004^{\star \star} \\
(0.001)\end{array}$ & $\begin{array}{c}0.005 \\
(0.005)\end{array}$ \\
\hline AVMTHSCH & $\begin{array}{l}0.106^{\star \star} \\
(0.011)\end{array}$ & $\begin{array}{l}0.108^{\star \star} \\
(0.020)\end{array}$ & $\begin{array}{l}0.090^{\star \star} \\
(0.022)\end{array}$ & $\begin{array}{c}0.085 \\
(0.057)\end{array}$ & $\begin{array}{l}0.123^{\star \star} \\
(0.018)\end{array}$ & $\begin{array}{c}0.049^{\star} \\
(0.023)\end{array}$ \\
\hline AVFTHSCH & $\begin{array}{c}0.018 \\
(0.011)\end{array}$ & $\begin{array}{c}0.004 \\
(0.018)\end{array}$ & $\begin{array}{c}0.045^{\star} \\
(0.023)\end{array}$ & $\begin{array}{c}0.032 \\
(0.056)\end{array}$ & $\begin{array}{c}0.029 \\
(0.018)\end{array}$ & $\begin{array}{c}-0.004 \\
(0.021)\end{array}$ \\
\hline AVINCOME ${ }^{\mathrm{a}}$ & $\begin{array}{c}0.024 \\
(0.030)\end{array}$ & $\begin{array}{l}0.086^{\star \star} \\
(0.029)\end{array}$ & $\begin{array}{c}-0.001 \\
(0.016)\end{array}$ & $\begin{array}{c}-0.006 \\
(0.077)\end{array}$ & $\begin{array}{c}-0.035 \\
(0.021)\end{array}$ & $\begin{array}{c}0.012^{\star} \\
(0.005)\end{array}$ \\
\hline RURAL & $\begin{array}{c}0.061^{\star} \\
(0.026)\end{array}$ & $\begin{array}{c}0.026 \\
(0.055)\end{array}$ & $\begin{array}{c}-0.079 \\
(0.090)\end{array}$ & $\begin{array}{l}-0.539^{\star \star} \\
(0.131)\end{array}$ & $\begin{array}{c}0.003 \\
(0.063)\end{array}$ & $\begin{array}{l}-0.193^{\star \star} \\
(0.069)\end{array}$ \\
\hline$\lambda^{\mathrm{a}}$ & $\begin{array}{c}-0.111 \\
(0.080)\end{array}$ & $\begin{array}{c}0.026 \\
(0.047)\end{array}$ & $\begin{array}{c}0.037 \\
(0.066)\end{array}$ & $\begin{array}{c}-0.558^{\star} \\
(0.226)\end{array}$ & $\begin{array}{c}-0.018 \\
(0.050)\end{array}$ & $\begin{array}{c}0.084^{\star} \\
(0.034)\end{array}$ \\
\hline CONSTANT & $\begin{array}{l}-1.822^{\star \star} \\
(0.064)\end{array}$ & $\begin{array}{l}-1.845^{\star \star} \\
(0.111)\end{array}$ & $\begin{array}{c}-1.789^{\star \star} \\
(0.194)\end{array}$ & $\begin{array}{c}0.377 \\
(0.885)\end{array}$ & $\begin{array}{l}-1.935^{\star \star} \\
(0.120)\end{array}$ & $\begin{array}{c}-0.950^{\star \star} \\
(0.318)\end{array}$ \\
\hline Number of individuals & 62684 & 30050 & 18442 & 2268 & 31249 & 14179 \\
\hline$R^{2}$ & 0.163 & 0.142 & 0.186 & 0.192 & 0.183 & 0.085 \\
\hline
\end{tabular}

${ }^{a}$ See text for definition of $\lambda$.

$\star \star$ Statistically significant at $1 \%, \star$ statistically significant at $5 \%$.

Note: Huber-White standard errors, adjusted for the clustering of students within schools, are presented in parentheses. Regional dummy variables were also included in regressions. 
Table 13. Mathematics achievement regressions with selectivity correction, 1997

\begin{tabular}{|c|c|c|c|c|c|c|}
\hline & \multicolumn{6}{|c|}{ Dependent variable: MATH } \\
\hline & $\begin{array}{l}\text { Public } \\
\text { DAEM }\end{array}$ & $\begin{array}{c}\text { Public } \\
\text { corporation }\end{array}$ & $\begin{array}{l}\text { Catholic } \\
\text { voucher }\end{array}$ & $\begin{array}{c}\text { Protestant } \\
\text { voucher }\end{array}$ & $\begin{array}{l}\text { Non- } \\
\text { religious } \\
\text { voucher }\end{array}$ & $\begin{array}{c}\text { Private } \\
\text { non- } \\
\text { voucher }\end{array}$ \\
\hline FEMALE & $\begin{array}{c}-0.047^{\star} \\
(0.021)\end{array}$ & $\begin{array}{c}-0.044 \\
(0.023)\end{array}$ & $\begin{array}{c}-0.031 \\
(0.029)\end{array}$ & $\begin{array}{c}-0.074 \\
(0.042)\end{array}$ & $\begin{array}{l}-0.050^{\star \star} \\
(0.018)\end{array}$ & $\begin{array}{c}0.046 \\
(0.026)\end{array}$ \\
\hline NATAM & $\begin{array}{l}-0.090^{\star \star} \\
(0.015)\end{array}$ & $\begin{array}{c}0.017 \\
(0.025)\end{array}$ & $\begin{array}{c}-0.071 \\
(0.040)\end{array}$ & $\begin{array}{c}-0.107 \\
(0.071)\end{array}$ & $\begin{array}{c}-0.016 \\
(0.023)\end{array}$ & $\begin{array}{c}-0.115 \\
(0.069)\end{array}$ \\
\hline MTHSCH & $\begin{array}{l}0.018^{\star \star} \\
(0.002)\end{array}$ & $\begin{array}{l}0.014^{\star \star} \\
(0.002)\end{array}$ & $\begin{array}{l}0.016^{\star \star} \\
(0.004)\end{array}$ & $\begin{array}{c}0.013 \\
(0.008)\end{array}$ & $\begin{array}{l}0.014^{\star \star} \\
(0.002)\end{array}$ & $\begin{array}{l}0.025^{\star \star} \\
(0.003)\end{array}$ \\
\hline MTHMISS & $\begin{array}{c}0.041 \\
(0.031)\end{array}$ & $\begin{array}{c}0.006 \\
(0.043)\end{array}$ & $\begin{array}{c}0.066 \\
(0.067)\end{array}$ & $\begin{array}{c}0.218 \\
(0.168)\end{array}$ & $\begin{array}{c}-0.003 \\
(0.046)\end{array}$ & $\begin{array}{l}0.280^{\star \star} \\
(0.089)\end{array}$ \\
\hline FTHSCH & $\begin{array}{l}0.010^{\star \star} \\
(0.002)\end{array}$ & $\begin{array}{l}0.005^{\star \star} \\
(0.002)\end{array}$ & $\begin{array}{l}0.010^{\star \star} \\
(0.003)\end{array}$ & $\begin{array}{c}0.002 \\
(0.009)\end{array}$ & $\begin{array}{l}0.007^{\star \star} \\
(0.002)\end{array}$ & $\begin{array}{l}0.016^{\star \star} \\
(0.003)\end{array}$ \\
\hline FTHMISS & $\begin{array}{c}-0.001 \\
(0.026)\end{array}$ & $\begin{array}{c}-0.048 \\
(0.032)\end{array}$ & $\begin{array}{c}0.063 \\
(0.056)\end{array}$ & $\begin{array}{c}-0.144 \\
(0.130)\end{array}$ & $\begin{array}{l}-0.101^{\star \star} \\
(0.036)\end{array}$ & $\begin{array}{c}0.092 \\
(0.079)\end{array}$ \\
\hline $\mathrm{INCOME}^{\mathrm{a}}$ & $\begin{array}{c}-0.005 \\
(0.008)\end{array}$ & $\begin{array}{c}-0.003 \\
(0.004)\end{array}$ & $\begin{array}{c}0.000 \\
(0.003)\end{array}$ & $\begin{array}{c}0.002 \\
(0.014)\end{array}$ & $\begin{array}{c}-0.004 \\
(0.003)\end{array}$ & $\begin{array}{c}0.006 \\
(0.003)\end{array}$ \\
\hline BOOKS1 & $\begin{array}{l}-0.080^{\star \star} \\
(0.012)\end{array}$ & $\begin{array}{c}-0.093^{\star \star} \\
(0.019)\end{array}$ & $\begin{array}{l}-0.133^{\star \star} \\
(0.037)\end{array}$ & $\begin{array}{c}-0.032 \\
(0.075)\end{array}$ & $\begin{array}{l}-0.106^{\star \star} \\
(0.023)\end{array}$ & $\begin{array}{c}-0.127 \\
(0.166)\end{array}$ \\
\hline BOOKS3 & $\begin{array}{l}0.072^{\star \star} \\
(0.014)\end{array}$ & $\begin{array}{l}0.071^{\star \star} \\
(0.016)\end{array}$ & $\begin{array}{c}0.026 \\
(0.026)\end{array}$ & $\begin{array}{c}0.072 \\
(0.065)\end{array}$ & $\begin{array}{l}0.076^{\star \star} \\
(0.018)\end{array}$ & $\begin{array}{c}0.057 \\
(0.045)\end{array}$ \\
\hline BOOKS4 & $\begin{array}{l}0.132^{\star \star} \\
(0.021)\end{array}$ & $\begin{array}{l}0.111^{\star \star} \\
(0.017)\end{array}$ & $\begin{array}{l}0.102^{\star \star} \\
(0.028)\end{array}$ & $\begin{array}{c}0.138^{\star} \\
(0.058)\end{array}$ & $\begin{array}{l}0.119^{\star \star} \\
(0.019)\end{array}$ & $\begin{array}{c}0.085 \\
(0.044)\end{array}$ \\
\hline BOOKS5 & $\begin{array}{c}0.192^{\star \star} \\
(0.028)\end{array}$ & $\begin{array}{l}0.153^{\star \star} \\
(0.024)\end{array}$ & $\begin{array}{c}0.122^{\star \star} \\
(0.035)\end{array}$ & $\begin{array}{c}0.092 \\
(0.083)\end{array}$ & $\begin{array}{c}0.174^{\star \star} \\
(0.024)\end{array}$ & $\begin{array}{c}0.110^{\star} \\
(0.049)\end{array}$ \\
\hline BOOKS6 & $\begin{array}{l}0.171^{\star \star} \\
(0.031)\end{array}$ & $\begin{array}{l}0.136^{\star \star} \\
(0.026)\end{array}$ & $\begin{array}{c}0.126^{\star \star} \\
(0.038)\end{array}$ & $\begin{array}{l}0.287^{\star \star} \\
(0.082)\end{array}$ & $\begin{array}{l}0.161^{\star \star} \\
(0.025)\end{array}$ & $\begin{array}{l}0.158^{\star \star} \\
(0.050)\end{array}$ \\
\hline BOOKS7 & $\begin{array}{l}0.198^{\star \star} \\
(0.038)\end{array}$ & $\begin{array}{l}0.137^{\star \star} \\
(0.033)\end{array}$ & $\begin{array}{l}0.203^{\star \star} \\
(0.043)\end{array}$ & $\begin{array}{c}0.229^{\star} \\
(0.098)\end{array}$ & $\begin{array}{l}0.214^{\star \star} \\
(0.033)\end{array}$ & $\begin{array}{c}0.186^{\star \star} \\
(0.051)\end{array}$ \\
\hline BOOKS8 & $\begin{array}{c}0.295^{\star \star} \\
(0.035)\end{array}$ & $\begin{array}{l}0.253^{\star \star} \\
(0.020)\end{array}$ & $\begin{array}{c}0.202^{\star \star} \\
(0.030)\end{array}$ & $\begin{array}{l}0.199^{\star \star} \\
(0.050)\end{array}$ & $\begin{array}{c}0.255^{\star \star} \\
(0.021)\end{array}$ & $\begin{array}{l}0.257^{\star \star} \\
(0.045)\end{array}$ \\
\hline PCTNATAM & $\begin{array}{c}-0.003^{\star \star} \\
(0.001)\end{array}$ & $\begin{array}{c}-0.002 \\
(0.003)\end{array}$ & $\begin{array}{c}-0.0001 \\
(0.004)\end{array}$ & $\begin{array}{c}-0.002 \\
(0.003)\end{array}$ & $\begin{array}{c}-0.0002 \\
(0.002)\end{array}$ & $\begin{array}{c}0.004 \\
(0.008)\end{array}$ \\
\hline AVMTHSCH & $\begin{array}{c}0.110^{\star \star} \\
(0.014)\end{array}$ & $\begin{array}{l}0.129^{\star \star} \\
(0.025)\end{array}$ & $\begin{array}{l}0.102^{\star \star} \\
(0.031)\end{array}$ & $\begin{array}{c}0.119 \\
(0.063)\end{array}$ & $\begin{array}{l}0.130^{\star \star} \\
(0.023)\end{array}$ & $\begin{array}{c}0.055 \\
(0.030)\end{array}$ \\
\hline AVFTHSCH & $\begin{array}{c}0.007 \\
(0.013)\end{array}$ & $\begin{array}{c}-0.035 \\
(0.024)\end{array}$ & $\begin{array}{c}0.031 \\
(0.030)\end{array}$ & $\begin{array}{c}-0.010 \\
(0.065)\end{array}$ & $\begin{array}{c}0.028 \\
(0.024)\end{array}$ & $\begin{array}{c}-0.021 \\
(0.029)\end{array}$ \\
\hline AVINCOME $^{\mathrm{a}}$ & $\begin{array}{c}0.060 \\
(0.043)\end{array}$ & $\begin{array}{l}0.142^{\star \star} \\
(0.044)\end{array}$ & $\begin{array}{c}0.029 \\
(0.020)\end{array}$ & $\begin{array}{c}-0.069 \\
(0.087)\end{array}$ & $\begin{array}{c}-0.009 \\
(0.026)\end{array}$ & $\begin{array}{l}0.027^{\star \star} \\
(0.008)\end{array}$ \\
\hline RURAL & $\begin{array}{l}0.187^{\star \star} \\
(0.033)\end{array}$ & $\begin{array}{c}0.128^{\star} \\
(0.061)\end{array}$ & $\begin{array}{c}0.029 \\
(0.113)\end{array}$ & $\begin{array}{c}-0.267^{\star} \\
(0.125)\end{array}$ & $\begin{array}{c}0.079 \\
(0.120)\end{array}$ & $\begin{array}{c}-0.188^{\star} \\
(0.087)\end{array}$ \\
\hline$\lambda^{\mathrm{a}}$ & $\begin{array}{c}-0.162 \\
(0.117)\end{array}$ & $\begin{array}{c}-0.034 \\
(0.066)\end{array}$ & $\begin{array}{c}0.062 \\
(0.087)\end{array}$ & $\begin{array}{c}-0.333 \\
(0.202)\end{array}$ & $\begin{array}{c}0.014 \\
(0.067)\end{array}$ & $\begin{array}{c}0.106^{\star} \\
(0.045)\end{array}$ \\
\hline CONSTANT & $\begin{array}{l}-1.594^{\star \star} \\
(0.077)\end{array}$ & $\begin{array}{l}-1.577^{\star \star} \\
(0.154)\end{array}$ & $\begin{array}{c}-1.729^{\star \star} \\
(0.285)\end{array}$ & $\begin{array}{c}-0.193 \\
(0.754)\end{array}$ & $\begin{array}{l}-1.949^{\star \star} \\
(0.153)\end{array}$ & $\begin{array}{c}-0.870 \\
(0.466)\end{array}$ \\
\hline Number of individuals & 62684 & 30050 & 18442 & 2268 & 31249 & 14179 \\
\hline$R^{2}$ & 0.124 & 0.124 & 0.167 & 0.149 & 0.161 & 0.083 \\
\hline
\end{tabular}

${ }^{a}$ See text for definition of $\lambda$.

$\star \star$ Statistically significant at $1 \%, \star$ statistically significant at $5 \%$.

Note: Huber-White standard errors, adjusted for the clustering of students within schools, are presented in parentheses. Regional dummy variables were also included in regressions. 
In the SPANISH regressions, coefficients on $\lambda_{i j}$ are negative for public DAEM, Protestant, and non-religious alternatives, although they are only statistically significant in the case of Protestant schools. Negative coefficients imply that unobservable characteristics of students in these school types tend to lower achievement. There are positive coefficients for public corporation, Catholic, and private non-voucher schools, although only the latter is statistically significant. The positive coefficients imply that student unobservables tend to increase achievement in these schools. The main results are duplicated in the MATH regressions. There is, for example, a negative and insignificant selectivity coefficient on public DAEM schools, and a positive and significant coefficient for private non-voucher schools.

What conclusions can be drawn from these results? The positive and significant coefficients for private non-voucher schools are consistent with evidence from other sources showing that private schools frequently select students with unobservable characteristics that may positively influence achievement. ${ }^{24}$ Point estimates of the other selectivity coefficients, while not precisely estimated, are generally suggestive of negative selection in public DAEM schools and positive selection in Catholic schools. Even so, the results do not provide enough evidence to convincingly reject the null hypothesis of no selection bias, especially in the case of public and private voucher schools. A possible solution would be to obtain a more complete set of instrumental variables - that is, variables that are correlated with private school choice, and uncorrelated with achievement. By so doing, one might diminish the amount of collinearity that the lambda term introduces to the achievement regressions, thus allowing coefficients and private school effects to be estimated with greater precision. Unfortunately, instrumental variables with these desirable properties are in short supply.

Whether or not the selectivity coefficients are statistically significant, the inclusion of these variables tends to alter other coefficient estimates. Thus, I recalculated the private school effects and standard errors using the new set of regression estimates. ${ }^{25}$ The results are presented in Tables 7 and 8 . With the exception of the Protestant alternative, accounting for selectivity reduces the advantage of other schools relative to the DAEM alternative. ${ }^{26}$ For example, the effect on Spanish achievement of private non-voucher schools declines from 0.46 to 0.12. The Catholic effect turns negative, while the public corporation and nonreligious effects become increasingly negative. However, most of these effects have quite large standard errors relative to their effects. The results are quite similar when mathematics is used as the dependent variable.

As before, private schools appear relatively more effective when corporation schools are used as the baseline. In most cases, however, accounting for selectivity reduces their relative effectiveness. The effect on Spanish of private non-voucher schools declines from 0.48 to 0.28 . The Catholic effect on Spanish declines slightly from 0.18 to 0.15 . In the case of non-religious schools, the selectivity correction leads to a slightly larger private school effect of 0.10 . This appears due to small positive selection among corporation students, and small negative selection among non-religious voucher students. But the new effect, like most of the others, is not much larger than its standard error.

\section{Summary and Conclusions}

This paper compares the academic achievement of eighth-grade students in six types of schools: public DAEM, public corporation, Catholic voucher, Protestant 
voucher, non-religious voucher, and private non-voucher schools. Controlling for individual and peer characteristics, the initial results suggested that the average public student would achieve highest in private non-voucher schools, relative to the public DAEM alternative. Achievement is around one-half of a standard deviation higher on the Spanish and mathematics tests. Catholic school effects, while still positive, are smaller in magnitude. Finally, non-religious voucher schools-by far the largest category of private schools - are somewhat less effective than DAEM schools and similarly effective to corporation schools.

Further attempts were made to control for unobserved student characteristics that could bias results. For public DAEM comparisons, controlling for selectivity served in every case to reduce the advantage of private schools; in fact, it only remained positive for private non-voucher students. When public corporation schools are used as the baseline for comparison, results are more favorable for private schools, although corporation schools make up a much smaller percentage of total public enrollments. Accounting for selectivity reduces private school effects, but they are still positive for Catholic and non-voucher private schools. Notwithstanding these results, the coefficients on the selectivity coefficients were rarely statistically significant, and the standard errors of the private schools effects were quite large. This prevents us from convincingly rejecting the hypothesis of no selection bias.

Many policy-makers are interested in the potential impact of large-scale voucher plans. In light of this, perhaps the most interesting finding of this paper is the lack of any consistent difference between student achievement in public and nonreligious voucher schools. Non-religious voucher schools are the largest category of private schooling in Chile, and the majority were created in direct response to the 1980 voucher reforms (most Catholic schools existed prior to the reform). But once student background and peer characteristics are taken into account, non-religious voucher schools produce no greater achievement than public schools, and may even produce slightly lower achievement. This finding is quite robust, and two pieces of evidence suggest that it may still be generous to private schools. First, this paper's attempts to correct for selection bias are suggestive, but not conclusive, that uncorrected estimates are biased in favor of private schools. Second, a convincing body of survey evidence shows that private schools often select students with unobserved characteristics that could positively influence achievement (Parry, 1996; Gauri, 1998).

In spite of these findings, enrollments in non-religious voucher schools rapidly increased throughout the 1980s. This begs the question of why families continued to choose these schools in large numbers, despite their comparable or even lower effectiveness. One possibility is that non-religious private schools excel at producing outcomes besides academic achievement. For example, they may provide a safe and orderly environment, promote important social and academic links to universities, or confer added prestige in a social milieu where 'private' is perceived as higher quality.

Another possibility is that private schools do, in fact, produce higher academic achievement, but that gains are rooted in the composition of school peer groups rather than the increased 'effort' of private school teachers and administrators. The previous empirical analyses controlled for the attributes of student peer groups. However, when controls for peer attributes were excluded, non-religious private schools appeared more effective because they enroll higher-SES students on average and students seem to benefit from exposure to high-SES peers. The relevant public/ 
private comparison for families may be that which does not correct for peer-group effects. When families choose among schooling options, they may care little whether higher achievement is delivered by higher-SES peers or harder-working private schools. The implication is that vouchers provided many middle-class Chilean families with the means to choose the 'right' peer group, rather than the 'best' school.

Finally, we must admit the possibility that the small differences in effectiveness between public and non-religious private schools are the result of almost 20 years of market competition that led to improvements in public schools. A private school advantage might have existed in the early years of the voucher plan, but disappeared in the 1990s as public schools responded to declining revenues by improving their effectiveness and perhaps eliminating the public/private gap in achievement. This is an open question that is being explored in other empirical research (McEwan \& Carnoy, 1999).

\section{Notes}

1. For reviews of the early studies (mainly using the 'High School and Beyond' data set in the US), see Haertel (1987) and Witte (1992). More recent reviews of the US literature include Neal (1998), Levin (1998), and Witte (1996). International studies include Jimenez and Lockheed (1995) and Kingdon (1996).

2. On Milwaukee's small-scale voucher experiment, see Greene et al. (1998), Rouse (1998), and Witte (1998). Recent voucher experiments have also been conducted in Dayton, OH, New York City, and Washington, DC (Peterson et al., 1998; Howell \& Peterson, 2000; Wolf et al., 2000).

3. For further details on the Chilean reforms, see Espinola (1993), Gauri (1998), Jofre (1988), and Parry (1997b).

4. DAEMs exist under the larger umbrella of the municipal bureaucracy and, as such, are governed by municipal rules. For instance, the head of the DAEM is required to be a teacher and he/she reports directly to the mayor. Employee contracts must conform to municipal regulations on hiring and salary scales. Corporations are non-profit organizations that are not subject to direct mayoral control, although the mayor does preside over a governing board. Their operations are generally subject to fewer regulations. In contrast to DAEMs, the corporation head is not required to be a teacher and corporation employees are not subjected to municipal rules regarding the hiring and remuneration of municipal employees. These features of corporations have led some to argue that they are more effective or efficient in the provision of educational services, although evidence on this issue is sparse. For further details, see Espinola (1993) or Parry (1997a).

5. Chilean law specifies a factor by which the base voucher is adjusted for students at every grade level. Furthermore, selected municipalities receive 'zone assignments' to compensate for high poverty or isolation. It should be noted, however, that adjustments are largely ad hoc and may not reflect true variation in educational costs. Since 1987, rural schools within municipalities have received upward adjustments. See Parry (1997a) for further details.

6. In 1996, 81 of Chile's 334 municipalities did not have a single privately run school, although these municipalities, mainly isolated and rural, account for a small percentage of total enrollments.

7. A general discussion of education production functions is given in Hanushek (1986). Researchers commonly include a pre-test as an independent variable in the production function. The intent of using a 'value-added' specification instead of a 'levels' specification is to focus attention on the growth in achievement that occurs in a particular time period, thus avoiding biases from incompletely measured variables over the entire length of a student's school attendance. Given the lack of pre-test data, the 'levels' specification is used.

8. In fact, one can test whether it is more appropriate to estimate a single equation (which allows only intercepts to vary across school types) or several equations (which allow slopes and intercepts to vary across school types). I tested this by estimating a single equation with three 
sets of variables: (1) five dummy variables indicating school type, (2) a full set of background controls, and (3) a full set of interactions between school type dummies and the control variables. I then tested the null hypothesis that the coefficients on the interaction terms were jointly equal to zero. Using a Wald test, this null hypothesis was easily rejected $(p<0.01)$, indicating that separate equations are justified.

9. For the standard errors, I use the formula in Murnane et al. (1985): $\left[\overline{\mathrm{X}}^{\prime}\left(\mathrm{V}^{\mathrm{J}}+V^{\mathrm{DAEM}}\right) \overline{\mathrm{X}}\right]^{1 / 2}$ where $V^{\mathrm{DAEM}}$ is the estimated variance-covariance matrix from the public DAEM regression and $\mathrm{V}^{\mathrm{j}}$ is the matrix from the comparison group's regression.

10. See Murnane et al. (1985) for a methodological exposition in the context of public/private school comparisons. For an empirical example, see Jimenez and Lockheed (1995).

11. For methodological expositions of Lee's approach, see Maddala (1983), Schmertmann (1994), and Vella (1998). For recent applications in education, see Argys et al. (1996) or Kingdon (1996).

12. Specifically, $v_{i j}$ is assumed to be independently and identically distributed, following a type I extreme value distribution (Maddala, 1983).

13. Even if the two sets of variables are equal, the model might still be identified on functional form, given that private school selection is a non-linear function of the variables. However, there is little theoretical basis for using an identical set of variables in both regressions, and doing so often leads to large standard errors in the second-step regression, and unreliable coefficient estimates (Vella, 1998). In empirical analyses that are not reported in this paper I did attempt this specification, and it yielded private school effects with extremely large standard errors.

14. Even the large urban areas of Chile are composed of multiple municipalities (the sprawling city Santiago consists of 51). Thus, the density variable provides a good measure of the relative availability of schooling opportunities in each student's neighborhood.

15. See McEwan and Carnoy (2000) for an analysis of fourth-grade data between 1990 and 1996.

16. See Evans et al. (1992) for further details. Evans et al. (1992) and Robertson and Symons (1996) are among the few empirical studies that treat peer-group variables as endogenous.

17. These estimates are smaller than those obtained with school-level data from 1990 to 1996 (McEwan \& Carnoy, 2000). However, the 1997 regressions make more detailed controls for student background variables, which may remove some bias from estimates.

18. Full regression results are available from the author.

19. See Evans and Schwab (1995). They include peer measures in an alternate specification, which does not substantially alter their estimates of private school effects. Also see Riddell's (1993) critique of the literature on private school comparisons in developing countries.

20. Evans and Schwab (1995), using data from 'High School and Beyond', do provide evidence that estimates of school racial composition constructed with individual data correlate well with measures reported at the school level.

21. Even the large urban areas of Chile are composed of multiple municipalities (the sprawling city Santiago consists of 51 municipalities). Thus, the density variable provides a good measure of the relative availability of schooling opportunities in each student's neighborhood.

22. Neal's (1997) study of Catholic school effectiveness in the US used a similar supply variable: the number of Catholic schools per square mile in each student's county. Neal (1997) and Evans and Schwab (1995) posit that two other variables are determinants of school choice, but not achievement: each student's Catholic religious status and the percentage of Catholic adherents in each county. In Chile, available data were insufficient to pursue this strategy. But were they to become available, it is doubtful that the variables would be useful. First, the vast majority of Chileans are Catholic, removing most useful variation. Second, a wide variety of schools, even public, teach religion and otherwise duplicate many practices of Catholic schools.

23. Models that use choice-specific variables are often referred to as conditional, rather than multinomial logits. For the likelihood functions and other methodological details, see Greene (1997), Hensher and Johnson (1981), McFadden (1974), or Maddala (1983).

24. Parry's (1996) random survey shows that $15 \%$ of public and $63 \%$ of private voucher schools in Santiago use one of several methods to select students for admission, including entrance examinations, interviews, and minimum grade requirements. Similarly, Gauri's (1998) random survey of Santiago households shows that $18 \%$ of public school students took an examination in order to enroll in their present school. For private voucher and private nonvoucher schools, the figures were 37 and $82 \%$, respectively. 
25. I conducted an additional specification check to assess the robustness of these results. I excluded DENSITY ${ }^{2}$ (but not DENSITY) from the multinomial logit, and re-calculated lambda and the estimates of private school effects. This did not alter the basic pattern of effects or standard errors that I report.

26. The effect of Protestant schools becomes wildly positive after correcting for selection bias. It is probable that collinearity induced by the selectivity term, in concert with the smaller Protestant sample, led to extreme movement in coefficient estimates.

\section{Acknowledgements}

This research was supported by a Spencer Fellowship for Research Related to Education and a Ford Foundation grant to Martin Carnoy. I am grateful to Martin Carnoy, Cristián Cox, Henry Levin, Susanna Loeb, Thomas Nechyba, and two anonymous referees for their helpful comments, without implicating them for my conclusions or errors. I also extend my thanks to Chile's Ministry of Education for its cooperation in providing the data.

\section{References}

Aedo, C. (1996) Calidad de la educación y elementos de mercado, Educación en Chile: Un Desafio de Calidad (Santiago, ENERSIS).

Aedo, C. (1998) Differences in schools and student performance in Chile, in: Savedoff, W. D. (Ed.) Organization Matters: Agency Problems in Health and Education in Latin America (Washington, DC, Johns Hopkins University Press).

Aedo, C. \& Larranaga, O. (1994) Sistemas de entrega de los servicios sociales: la experiencia chilena, in Aedo, C. \& Larranaga, O. (Eds) Sistema de Entrega de los Servicios Sociales: Una Agenda para la Reforma (Washington, DC, Banco Interamericano de Desarrollo).

Argys, L. M., Rees, D. I. \& Brewer, D. J. (1996) Detracking America's schools: equity at zero cost?, Fournal of Policy Analysis and Management, 15, pp. 623-645.

Cox, D. \& Jimenez, E. (1990) The relative effectiveness of private and public schools: evidence from two developing countries, fournal of Development Economics, 34, pp. 99-121.

Espinola, V. (1993) The educational reform of the military regime in Chile: the system's response to competition, choice, and market relations, unpublished $\mathrm{PhD}$ Dissertation (University of Wales, UK).

Evans, W. N., Oates, W. E. \& Schwab, R. M. (1992) Measuring peer group effects: a study of teenage behavior, fournal of Political Economy, 100, pp. 966-991.

Evans, W. N. \& Schwab, R. M. (1995) Finishing high school and starting college: do Catholic schools make a difference?, Quarterly fournal of Economics, 10, pp. 941-974.

Gauri, V. (1998) School Choice in Chile: Two Decades of Educational Reform (Pittsburgh, PA, University of Pittsburgh Press).

Greene, W. H. (1997) Econometric Analysis, 3rd Edn (Upper Saddle River, NJ, Prentice Hall).

Greene, J. P., Peterson, P. E. \& Du, J. (1998) School choice in Milwaukee: a randomized experiment, in: Peterson, P. E. \& Hassel, B. C. (Eds) Learning from School Choice (Washington, DC, Brookings Institution Press).

Haertel, E. H. (1987) Comparing public and private schools using longitudinal data from the HSB study, in: Haertel, E. H., James, T. \& Levin, H. M. (Eds) Comparing Public and Private Schools, Vol. 2 (New York, The Falmer Press).

Hanushek, E. A. (1986) The economics of schooling: production and efficiency in public schools, fournal of Economic Literature, 24, pp. 1141-1177.

Heckman, J. J. (1979) Sample selection bias as a specification error, Econometrica, 47, pp. 153-161.

Hensher, D. A. \& Johnson, L. W. (1981) Applied Discrete-Choice Modelling (London, Croom Helm).

Howell, W. G. \& Peterson, P. E. (2000) School choice in Dayon, Ohio: an evaluation after one year, Program on Education Policy and Governance (Cambridge, MA, Harvard University).

Jimenez, E. \& Lockheed, M. E. (1995) Public and private secondary education in developing countries: a comparative study, World Bank Discussion Paper 309 (Washington, DC, World Bank). 
Jofre, G. (1988) El sistema de subvenciones en educación: La experiencia chilena. Estudios Públicos.

Kingdon, G. (1996) The quality and efficiency of private and public education: a case-study of urban India, Oxford Bulletin of Economics and Statistics, 58, pp. 57-82.

Lee, L.-F. (1983) Generalized econometric models with selectivity, Econometrica, 51, pp. 507-512.

Levin, H. M. (1998) Educational vouchers: effectiveness, choice, and costs, fournal of Policy Analysis and Management, 17, pp. 373-391.

Maddala, G. S. (1983) Limited-Dependent and Qualitative Variables in Econometrics (Cambridge, Cambridge University Press).

McEwan, P. J. \& Carnoy, M. (2000) The effectiveness and efficiency of private schools in Chile's voucher system, Educational Evaluation and Policy Analysis, 33, pp. 213-239.

McEwan, P. J. \& Carnoy, M. (1999) The impact of competition on public school quality: longitudinal evidence from Chile's voucher system, unpublished manuscript (Stanford University).

McFadden, D. (1974) Conditional logit analysis of qualitative choice behavior, in: Zarembka, P. (Ed.) Frontiers in Econometrics (New York, Academic Press).

Murnane, R. J., Newstead, S. \& Olsen, R. J. (1985) Comparing public and private schools: the puzzling role of selection bias, fournal of Business and Economic Statistics, 3, pp. 23-35.

Neal, D. (1997) The effects of Catholic secondary schooling on educational achievement, fournal of Labor Economics, 15, pp. 98-123.

Neal, D. (1998) What have we learned about the benefits of private schooling?, Federal Reserve Bank of New York Policy Review, 4, pp. 79-86.

Parry, T. R. (1996) Will pursuit of higher quality sacrifice equal opportunity in education?, An analysis of the education voucher system in Santiago, Social Science Quarterly, 77, pp. 821-841.

Parry, T. R. (1997a) Achieving balance in decentralization: a case study of education decentralization in Chile, World Development, 25, pp. 211-225.

Parry, T. R. (1997b) Decentralization and privatization: education policy in Chile, fournal of Public Policy, 17, pp. 107-133.

Parry, T. R. (1997c) Theory meets reality in the education voucher debate: some evidence from Chile, Education Economics, 5, pp. 307-331.

Peterson, P. E., Myers, D. \& Howell, W. G. (1998) An evaluation of the New York City school choice scholarships program: the first year, Program on Education Policy and Governance (Cambridge, MA, Harvard University).

Riddell, A. R. (1993) The evidence on public/private educational trade-offs in developing countries, International fournal of Educational Development, 13, pp. 373-386.

Robertson, D. \& Symons, J. (1996) Do peer groups matter? Peer group versus schooling effects on academic achievement, Discussion Paper 311 (London, London School of Economics Centre for Economic Performance).

Rouse, C. E. (1998) Private school vouchers and student achievement: an evaluation of the Milwaukee parental choice program, Quarterly fournal of Economics, 113, pp. 553-602.

Schmertmann, C. (1994) Selectivity bias correction methods in polychotomous sample selection models, fournal of Econometrics, 60, pp. 101-132.

Vella, F. (1998) Estimating models with sample selection bias: a survey, fournal of Human Resources, 33, pp. 127-169.

Witte, J. F. (1992) Private school versus public school achievement: are there findings that should affect the educational choice debate?, Economics of Education Review, 11, pp. 371-394.

Witte, J. F. (1996) School choice and student performance, in: Ladd, H. F. (Ed.) Holding Schools Accountable: Performance-Based Reform in Education (Washington, DC, The Brookings Institution).

Witte, J. F. (1998) The Milwaukee voucher experiment, Educational Evaluation and Policy Analysis, 20, pp. 229-251.

Wolf, P. J., Howell, W. G. \& Peterson, P. E. (2000) School choice in Washington, DC: an evaluation after one year, Program on Education Policy and Governance (Cambridge, MA, Harvard University). 